\title{
Multi-dimensional metric approximation by primitive points
}

\author{
S. G. DANI, Michel LAURENT, Arnaldo NOGUEIRA
}

॥1

\begin{abstract}
We consider diophantine inequalities of the form $|\Theta \mathbf{q}+\mathbf{p}-\mathbf{y}| \leq \psi(|\mathbf{q}|)$, with $\Theta \in \operatorname{Mat}_{n, m}(\mathbb{R}), \mathbf{y} \in \mathbb{R}^{n}$, where $m, n \in \mathbb{N}$, and $\psi$ is a function on $\mathbb{N}$ with positive real values, seeking integral solutions $\mathbf{v}=(\mathbf{q}, \mathbf{p})^{t}$ for which the restriction of $\mathbf{v}$ to the components of a given partition $\pi$ are primitive integer points. In this setting, we establish metrical statements in the style of the Khintchine-Groshev Theorem. Similar solutions are considered for the doubly metrical inequality $|\Theta \mathbf{q}+\Phi \mathbf{p}-\mathbf{y}| \leq$ $\psi(|\mathbf{q}|)$, with $\Phi \in \operatorname{Mat}_{n, n}(\mathbb{R})$ (other notation as before). The results involve the conditions that $x \mapsto x^{m-1} \psi(x)^{n}$ be non-increasing, and that the components of $\pi$ have at least $n+1$ elements each.
\end{abstract}

\section{Introduction}

For $d \in \mathbb{N}$ we view $\mathbb{R}^{d}$ as the space of $d$-rowed column vectors $\mathbf{v}=\left(v_{1}, \ldots, v_{d}\right)^{t}$, $v_{1}, \ldots, v_{d} \in \mathbb{R}$, (the $t$ stands for transpose). For $\mathbf{v} \in \mathbb{R}^{d}$ denote by $|\mathbf{v}|$ the supremum norm in $\mathbb{R}^{d}$. When $\mathbf{w} \in \mathbb{R}^{m}$ and $\mathbf{v} \in \mathbb{R}^{n}$, we conventionally write as $(\mathbf{w}, \mathbf{v})^{t}$ the vector $\left(\begin{array}{l}\mathbf{w} \\ \mathbf{v}\end{array}\right)$ in $\mathbb{R}^{m+n}$. Also, for $m, n \in \mathbb{N}$, we denote by $\operatorname{Mat}_{n, m}(\mathbb{R})$ the vector space of $n \times m$ matrices with real entries. A matrix $X \in \operatorname{Mat}_{n, m+n}(\mathbb{R})$ will be expressed in the form $X=(\Theta, \Phi)$ with $\Theta \in \operatorname{Mat}_{n, m}(\mathbb{R})$ and $\Phi \in \operatorname{Mat}_{n, n}(\mathbb{R})$.

We denote by $P\left(\mathbb{Z}^{d}\right)$ the set of primitive points in $\mathbb{Z}^{d}$, that is, the set of integer $d$-tuples $\mathbf{v}=\left(v_{1}, \ldots, v_{d}\right)^{t}$ with $\operatorname{gcd}\left(v_{1}, \ldots, v_{d}\right)=1$. For any subset $\sigma=\left\{i_{1}, \ldots, i_{\nu}\right\}$ of $\{1, \ldots, d\}$ having $\nu \geq 2$ elements, we denote by $P(\sigma)$ the set of integer points $\mathbf{v}=$

2010 Mathematics Subject Classification: 11J20, 37A17.

Key words: diophantine approximation, metrical number theory, primitive points, ergodic theory. 
$\left(v_{1}, \ldots, v_{d}\right)^{t}$ such that $\operatorname{gcd}\left(v_{i_{1}}, \ldots, v_{i_{\nu}}\right)=1$. Let $\pi$ be a partition of $\{1, \ldots, d\}$ formed by a family of subsets $\pi_{j}$ each one with at least two elements. Then, we denote by $P(\pi)$ the set of integer points $\mathbf{v} \in \mathbb{Z}^{d}$ such that $\mathbf{v} \in P\left(\pi_{j}\right)$ for all components $\pi_{j}$.

Our goal is to refine classical results on metrical diophantine approximation for systems of linear or affine inequalities, in the style of the Khintchine-Groshev Theorem, with stipulation of additional constraints on the solutions being sought, involving coprimality; if $\mathbf{v}$ stands for the vector of (integral) solution, these conditions are expressed in the form $\mathbf{v} \in P(\pi), \pi$ a partition of $\{1, \ldots, m+n\}$. The following theorem refines, in this respect, results due to Cassels for $m=1$ and Sprindzuck for $m \geq 2$ (Theorem II of Chapter VII in [6] and Theorem 15 in Chapter I of [19]); it may be noted however that we need a monotonicity assumption on the approximating function $\psi$, as seen in the statement below, though both of the theorems mentioned are proved without any monotonicity condition.

Theorem 1.1. Let $n, m \in \mathbb{N}$ and $\pi$ be a partition of $\{1, \ldots, m+n\}$ such that every component of $\pi$ has at least $n+1$ elements. Let $\psi: \mathbb{N} \rightarrow(0, \infty)$ be a function such that the mapping $x \mapsto x^{m-1} \psi(x)^{n}$ is non-increasing. If

$$
\sum_{j \geq 1} j^{m-1} \psi(j)^{n}=\infty,
$$

then for almost every pair $(\Theta, \mathbf{y}) \in \operatorname{Mat}_{n, m}(\mathbb{R}) \times \mathbb{R}^{n}$, there exist infinitely many points $(\mathbf{q}, \mathbf{p})^{t} \in P(\pi)$ such that

$$
|\Theta \mathbf{q}+\mathbf{p}-\mathbf{y}| \leq \psi(|\mathbf{q}|) .
$$

Conversely, if the series $\sum_{j \geq 1} j^{m-1} \psi(j)^{n}$ converges, then for almost every pair $(\Theta, \mathbf{y}) \in \operatorname{Mat}_{n, m}(\mathbb{R}) \times \mathbb{R}^{n}$, there exist only finitely many integer points $(\mathbf{q}, \mathbf{p})^{t} \in \mathbb{Z}^{m+n}$ for which (1.1) holds.

In the case $m=n=1$, Theorem 1.1 was proved in [13, under the additional assumption on the growth of the function $\psi$, that $\psi(2 \ell) \gg \psi(\ell)$, namely $\psi(2 \ell) / \psi(\ell)$ bounded below by a positive constant, for all $l \in \mathbb{N}$; see Theorem 2 in [13].

Theorem 1.1 is a doubly metrical statement in the sense that it concerns pairs $(\Theta, \mathbf{y})$. We expect that the same conclusion should hold for any given $\mathbf{y} \in \mathbb{R}^{n}$ (not just for almost all $\mathbf{y}$ ) for almost all $\Theta$; this is suggested by the fact that the corresponding statement without the coprimality condition $(\mathbf{q}, \mathbf{p})^{t} \in P(\pi)$ can be deduced, for every fixed $\mathbf{y} \in \mathbb{R}^{n}$, from the work [17] of Schmidt; the latter also provides further information, such as counting formulas for the number of solutions. Further metrical results of inhomogeneous approximation can also be found in [5]. For the case of $\mathbf{y}=0$, viz. the homogeneous case, we are able to establish such 
a statement, thus providing a refinement of the well-known Khintchine-Groshev Theorem [3, 9, 11].

Theorem 1.2. Let $n, m, \pi$ and $\psi$ be as in Theorem 1.1. If $\sum_{j \geq 1} j^{m-1} \psi(j)^{n}=\infty$ then for almost every $\Theta \in \operatorname{Mat}_{n, m}(\mathbb{R})$, there exist infinitely many points $(\mathbf{q}, \mathbf{p})^{t} \in$ $P(\pi)$ such that

$$
|\Theta \mathbf{q}+\mathbf{p}| \leq \psi(|\mathbf{q}|)
$$

Conversely, if $\sum_{j \geq 1} j^{m-1} \psi(j)^{n}<\infty$ then for almost every $\Theta \in \operatorname{Mat}_{n, m}(\mathbb{R})$, there exist only finitely many $(\mathbf{q}, \mathbf{p})^{t} \in \mathbb{Z}^{m+n}$ for which (1.2) holds.

Theorems 1.1 and 1.2 concern 'normalized' systems of $n$ linear forms $\Theta \mathbf{q}+\mathbf{p}$ with rank $n$, meaning that the coefficient matrix of $\mathbf{p}$ is the identity. For general systems with free coefficients, we obtain the expected metrical statement for any fixed $\mathbf{y}$ :

Theorem 1.3. Let $n, m, \pi$ and $\psi$ be as in Theorem 1.1. If $\sum_{j>1} j^{m-1} \psi(j)^{n}=\infty$ then for every $\mathbf{y} \in \mathbb{R}^{n}$ and for almost every matrix $X=(\Theta, \Phi) \in \operatorname{Mat}_{n, m+n}(\mathbb{R})$, there exist infinitely many points $(\mathbf{q}, \mathbf{p})^{t} \in P(\pi)$ such that

$$
|\Theta \mathbf{q}+\Phi \mathbf{p}-\mathbf{y}| \leq \psi(|\mathbf{q}|)
$$

Conversely, if $\sum_{j \geq 1} j^{m-1} \psi(j)^{n}<\infty$ then for almost every $X=(\Theta, \Phi) \in \operatorname{Mat}_{n, m+n}(\mathbb{R})$, there exist only finitely many $(\mathbf{q}, \mathbf{p})^{t} \in \mathbb{Z}^{m+n}$ for which (1.3) holds.

It would be worthwhile to illustrate our theorems with two special cases. The first one may be viewed as an extension of Theorem 2 of [13] to simultaneous approximation, as well as an inhomogeneous version of Gallagher's result [8]:

Corollary 1.4. Let $n$ be a positive integer and let $\psi: \mathbb{N} \mapsto \mathbb{R}^{+}$be a non-increasing function such that $\sum_{j \geq 1} \psi(j)^{n}=\infty$. For almost every real $2 n$-tuple $\left(\theta_{1}, \ldots, \theta_{n}, y_{1}, \ldots, y_{n}\right)$ there exist infinitely many integer points $\left(q, p_{1}, \ldots, p_{n}\right)$ such that

$$
\operatorname{gcd}\left(q, p_{1}, \ldots, p_{n}\right)=1 \text { and } \max _{1 \leq i \leq n}\left(\left|q \theta_{i}+p_{i}-y_{i}\right|\right) \leq \psi(|q|) \text {. }
$$

Proof. This is the special case of Theorem 1.1, with $m=1$ and $\pi$ the trivial partition of $\{1, \ldots, n+1\}$ (with only one component).

The following is an application to linear approximation; it may be noted that even the homogeneous case with $y=0$ is not obvious when $k \geq 2$.

Corollary 1.5. Let $k$ be a positive integer and let $\psi: \mathbb{N} \mapsto \mathbb{R}^{+}$be a function such that the mapping $x \mapsto x^{2 k-2} \psi(x)$ is non-increasing and $\sum_{j \geq 1} j^{2 k-2} \psi(j)=\infty$. For 
every real number $y$ and almost every real linear form $L(\mathbf{x})=\alpha_{1} x_{1}+\cdots+\alpha_{2 k} x_{2 k}$ in $2 k$ variables, there exist infinitely many integer points $\left(q_{1}, \ldots, q_{2 k}\right)$ such that

$$
\operatorname{gcd}\left(q_{2 i-1}, q_{2 i}\right)=1 \text { for all } i=1, \ldots, k \text { and }\left|L\left(q_{1}, \ldots, q_{2 k}\right)-y\right| \leq \psi\left(\max _{1 \leq i \leq 2 k-1}\left|q_{i}\right|\right) \text {. }
$$

Proof. This is the special case of Theorem 1.3, with $m=2 k-1, n=1$ and $\pi$ the partition as $\{1, \ldots, 2 k\}=\coprod_{j=1}^{k}\{2 j-1,2 j\}$ into pairs of indices.

The method of our proofs of Theorems 1.1 and 1.2 involves a combination of standard methods in the metrical theory of diophantine approximation together with certain new $0-1$ laws. A key observation is that the set $P(\pi) \subseteq \mathbb{Z}^{m+n}$ is invariant under the action (usual) of a certain subgroup $\Gamma_{\pi}$ of $S L(m+n, \mathbb{Z}$ ), whose action on $\operatorname{Mat}_{n, m+n}(\mathbb{R})$ by right multiplication is ergodic whenever each component of the partition $\pi$ has at least $n+1$ elements. Corresponding to Theorems 1.1 and 1.2 we define in a natural way certain subsets of $\operatorname{Mat}_{n, m+n}(\mathbb{R})$ which are $\Gamma_{\pi}$-invariant. The ergodicity then implies the desired 0-1 laws for the subsets; the details involved

are described in Section 2. The proofs of Theorems 1.1 and 1.2 then proceed along the lines of that of the Khintchine-Groshev Theorem, following the same steps as [3]. The main ingredients are adapted in Sections 4 and 5 to our present framework involving points in $P(\pi)$. Section 3 is devoted to obtain an extension of a classical lemma due to Cassels that is needed in the proofs of the theorems. Theorem 1.3 readily follows as a consequence of Theorems 1.1 and 1.2, it may be worthwhile to note here that alternatively Theorem 1.1 and 1.2 could be deduced after proving Theorem 1.3 first.

\section{A general zero-one law}

Let $\mathfrak{O}$ be an infinite subset of $\mathbb{Z}^{m+n}$ and $\psi: \mathbb{N} \rightarrow(0, \infty)$ be a non-increasing function. We introduce the following sets depending on $\mathfrak{O}$ and $\psi$, for any $\mathbf{y} \in \mathbb{R}^{n}$. For $\mathbf{v}=(\mathbf{q}, \mathbf{p})^{t} \in \mathbb{Z}^{m+n}$ let us consider the 'strip'

$$
S_{\mathbf{v}}(\psi, \mathbf{y})=\left\{(\Theta, \Phi) \in \operatorname{Mat}_{n, m+n}(\mathbb{R}):|\Theta \mathbf{q}+\Phi \mathbf{p}-\mathbf{y}| \leq \psi(|\mathbf{q}|)\right\}
$$

For $(\Theta, \Phi) \in \operatorname{Mat}_{n, m+n}(\mathbb{R})$ let

$$
\mathfrak{O}(\Theta, \Phi)=\left\{\mathbf{v} \in \mathfrak{O}:(\Theta, \Phi) \in S_{\mathbf{v}}(\psi, \mathbf{y})\right\}
$$

and define

$$
\mathcal{G}_{\mathfrak{O}}(\psi, \mathbf{y})=\left\{(\Theta, \Phi) \in \operatorname{Mat}_{n, m+n}(\mathbb{R}): \mathfrak{O}(\Theta, \Phi) \text { is infinite }\right\}
$$


We shall be concerned with the Lebesgue measure of $\mathcal{G}_{P(\pi)}(\psi, \mathbf{y})$, and other analogous sets (with $\pi$ as in the theorems). Towards that end we first consider a related class of sets defined as follows. For any $l \in \mathbb{N}$ let $\psi_{l}$ be the function defined by $\psi_{l}(j)=\psi(l j)$ for all $j \in \mathbb{N}$ and set

$$
\mathcal{G}_{\mathfrak{O}}^{\prime}(\psi, \mathbf{y})=\bigcap_{l \in \mathbb{N}} \mathcal{G}_{\mathfrak{O}}\left(\psi_{l}, \mathbf{y}\right)
$$

We now describe certain conditions under which the set $\mathcal{G}_{\mathfrak{O}}^{\prime}(\psi, \mathbf{y})$ satisfies the $0-1$ law, namely it is either a null set (a set of measure 0) or a full set (complement of a set of measure 0 ), with respect to the Lebesgue measure on $\operatorname{Mat}_{n, m+n}(\mathbb{R})$.

In this respect we consider subsets $\mathfrak{O}$ which are orbits of a subgroup $\Gamma$ of $S L(m+$ $n, \mathbb{Z})$. We note that on $\operatorname{Mat}_{n, m+n}(\mathbb{R})$ there is an action of $S L(m+n, \mathbb{Z})$ by (matrix) multiplication on the right. We shall be interested in the case when the induced action of $\Gamma$ is ergodic with respect to the Lebesgue measure, viz. every $\Gamma$-invariant subset is either a null set or a full set.

Proposition 2.1. Let $\mathfrak{O}$ be an orbit of a subgroup $\Gamma$ of $S L(m+n, \mathbb{Z})$ whose action on $\operatorname{Mat}_{n, m+n}(\mathbb{R})$ is ergodic, and $\psi$ be as above. Then for every $\mathbf{y} \in \mathbb{R}^{n}, \mathcal{G}_{\mathfrak{O}}^{\prime}(\psi, \mathbf{y})$ is either a null set or a full set.

Proof. We show that $\mathcal{G}_{\mathfrak{O}}^{\prime}(\psi, \mathbf{y}) \bigcap\left(\operatorname{Mat}_{n, m}(\mathbb{R}) \times G L(n, \mathbb{R})\right)$ is invariant under the $\Gamma$ action. Since $G L(n, \mathbb{R})$ is a full set in $\operatorname{Mat}_{n, n}(\mathbb{R})$ the ergodicity condition would then imply that $\mathcal{G}_{\mathfrak{O}}^{\prime}(\psi, \mathbf{y})$ is either a null set or a full set, thus proving the proposition.

Let $(\Theta, \Phi) \in \mathcal{G}_{\mathfrak{O}}^{\prime}(\psi, \mathbf{y})$, with $\Phi \in G L(n, \mathbb{R})$, and $\gamma \in \Gamma$ be given. Let $(\Theta, \Phi) \gamma^{-1}=$ $\left(\Theta^{\prime}, \Phi^{\prime}\right)$; to prove the proposition it suffices to show that $\left(\Theta^{\prime}, \Phi^{\prime}\right) \in \mathcal{G}_{\mathfrak{O}}\left(\psi_{l}, \mathbf{y}\right)$ for all $l \in \mathbb{N}$. Let $l \in \mathbb{N}$ be given. Choose $a \in \mathbb{N}$ greater than $(m+n)|\gamma| \max \left(1,2 m n\left|\Phi^{-1}\right||\Theta|\right)$. As $(\Theta, \Phi)$ belongs to $\mathcal{G}_{\mathfrak{O}}\left(\psi_{a l}, \mathbf{y}\right)$, there exist infinitely many $\mathbf{v}=(\mathbf{q}, \mathbf{p})^{t} \in \mathfrak{O}$ such that $(\Theta, \Phi) \in S_{\mathbf{v}}\left(\psi_{a l}, \mathbf{y}\right)$. In other words, we have

$$
|\Theta \mathbf{q}+\Phi \mathbf{p}-\mathbf{y}| \leq \psi(a l|\mathbf{q}|)
$$

for these $\mathbf{v}$. Observe that we have the bounds

$|\mathbf{p}| \leq n\left|\Phi^{-1}\right||\Phi \mathbf{p}| \leq n\left|\Phi^{-1}\right|(|\Theta \mathbf{q}-\mathbf{y}|+\psi(a l|\mathbf{q}|)) \leq n\left|\Phi^{-1}\right|(m|\Theta||\mathbf{q}|+|\mathbf{y}|+\psi(a l|\mathbf{q}|))$,

and hence $|\mathbf{p}| \leq c|\mathbf{q}|$ for $c>m n\left|\Phi^{-1}\right||\Theta|$, when $|\mathbf{q}|$ is sufficiently large. Now put $\gamma(\mathbf{q}, \mathbf{p})^{t}=\left(\mathbf{q}^{\prime}, \mathbf{p}^{\prime}\right)^{t}$. We deduce from the above upper bounds the estimate

$$
\left|\mathbf{q}^{\prime}\right| \leq\left|\left(\mathbf{q}^{\prime}, \mathbf{p}^{\prime}\right)^{t}\right| \leq(m+n)|\gamma|\left|(\mathbf{q}, \mathbf{p})^{t}\right| \leq a|\mathbf{q}|,
$$

when $|\mathbf{q}|$ is sufficiently large. Write

$$
\Theta^{\prime} \mathbf{q}^{\prime}+\Phi^{\prime} \mathbf{p}^{\prime}=\left((\Theta, \Phi) \gamma^{-1}\right)\left(\gamma(\mathbf{q}, \mathbf{p})^{t}\right)=(\Theta, \Phi)(\mathbf{q}, \mathbf{p})^{t}=\Theta \mathbf{q}+\Phi \mathbf{p}
$$


Hence $\left|\Theta^{\prime} \mathbf{q}^{\prime}+\Phi^{\prime} \mathbf{p}^{\prime}-\mathbf{y}\right|=|\Theta \mathbf{q}+\Phi \mathbf{p}-\mathbf{y}| \leq \psi(a l|\mathbf{q}|)$. As $\left|\mathbf{q}^{\prime}\right| \leq a|\mathbf{q}|$, and $\psi$ is non-decreasing, we have $\psi(a l|\mathbf{q}|)=\psi_{l}(a|\mathbf{q}|) \leq \psi_{l}\left(\left|\mathbf{q}^{\prime}\right|\right)$, and thus $\left|\Theta^{\prime} \mathbf{q}^{\prime}+\Phi^{\prime} \mathbf{p}^{\prime}-\mathbf{y}\right| \leq$ $\psi_{l}\left(\left|\mathbf{q}^{\prime}\right|\right)$. Noting that $\left(\mathbf{q}^{\prime}, \mathbf{p}^{\prime}\right)^{t} \in \mathfrak{O}$, this shows that $(\Theta, \Phi) \gamma^{-1}=\left(\Theta^{\prime}, \Phi^{\prime}\right) \in \mathcal{G}_{\mathfrak{O}}\left(\psi_{l}, \mathbf{y}\right)$. As noted above this proves the proposition.

We next specialise to the 'normalized systems', where $\Phi=I_{n}$, the identity matrix in $\operatorname{Mat}_{n, n}(\mathbb{R})$. Put, with notation $\mathcal{G}_{\mathfrak{O}}(\psi, \mathbf{y})$ as in $(2.1)$,

$$
\mathcal{E}_{\mathfrak{D}}(\psi, \mathbf{y})=\left\{\Theta \in \operatorname{Mat}_{n, m}(\mathbb{R}):\left(\Theta, I_{n}\right) \in \mathcal{G}_{\mathfrak{O}}(\psi, \mathbf{y})\right\}
$$

and let

$$
\mathcal{E}_{\mathfrak{O}}(\psi)=\left\{(\Theta, \mathbf{y}) \in \operatorname{Mat}_{n, m}(\mathbb{R}) \times \mathbb{R}^{n}: \Theta \in \mathcal{E}_{\mathfrak{O}}(\psi, \mathbf{y})\right\}
$$

thus $\mathcal{E}_{P(\pi)}(\psi)$ is the set of all pairs $(\Theta, \mathbf{y}) \in \operatorname{Mat}_{n, m}(\mathbb{R}) \times \mathbb{R}^{n}$ satisfying (1.1). We now define correspondingly

$$
\mathcal{E}_{\mathfrak{O}}^{\prime}(\psi, \mathbf{y})=\bigcap_{l \in \mathbb{N}}\left(\bigcup_{\kappa \in \mathbb{N}} \mathcal{E}_{\mathfrak{O}}\left(\kappa \psi_{l}, \mathbf{y}\right)\right) \quad \text { and } \quad \mathcal{E}_{\mathfrak{D}}^{\prime}(\psi)=\bigcap_{l \in \mathbb{N}}\left(\bigcup_{\kappa \in \mathbb{N}} \mathcal{E}_{\mathfrak{O}}\left(\kappa \psi_{l}\right)\right) \text {. }
$$

Proposition 2.2. Let $\mathfrak{O}$ and $\psi$ be as in Proposition 2.1. Then $\mathcal{E}_{\mathfrak{D}}^{\prime}(\psi)$ is either a null set or a full set.

Proof. Let us introduce the larger set

$$
\mathcal{G}_{\mathfrak{O}}^{\prime \prime}(\psi, \mathbf{y})=\bigcap_{l \in \mathbb{N}}\left(\bigcup_{\kappa \in \mathbb{N}} \mathcal{G}_{\mathfrak{O}}\left(\kappa \psi_{l}, \mathbf{y}\right)\right)
$$

containing $\mathcal{G}_{\mathfrak{O}}^{\prime}(\psi, \mathbf{y})$. Arguing as in the proof of Proposition 2.1, we observe that $\mathcal{G}_{\mathfrak{O}}^{\prime \prime}(\psi, \mathbf{y}) \bigcap\left(\operatorname{Mat}_{n, m}(\mathbb{R}) \times G L(n, \mathbb{R})\right)$ is as well invariant under the $\Gamma$-action. It follows that $\mathcal{G}_{\mathfrak{O}}^{\prime \prime}(\psi, \mathbf{y})$ is either a null set or a full set for every fixed $\mathbf{y} \in \mathbb{R}^{n}$. Note now that the set of $\mathbf{y} \in \mathbb{R}^{n}$ for which $\mathcal{G}_{\mathfrak{O}}^{\prime \prime}(\psi, \mathbf{y})$ is of full Lebesgue measure is invariant under the action of $G L(n, \mathbb{R})$ by (matrix) left multiplication. Indeed, if $(\Theta, \Phi) \in \mathcal{G}_{\mathfrak{D}}\left(\kappa \psi_{l}, \mathbf{y}\right)$, for any $g \in G L(n, \mathbb{R})$ we have

$$
|g \Theta \mathbf{q}+g \Phi \mathbf{p}+g \mathbf{y}|=|g(\Theta \mathbf{q}+\Phi \mathbf{p}+\mathbf{y})| \leq n|g||\Theta \mathbf{q}+\Phi \mathbf{p}+\mathbf{y}| \leq n|g| \kappa \psi_{l}(|\mathbf{q}|)
$$

for infinitely many $(\mathbf{q}, \mathbf{p})^{t} \in \mathfrak{O}$. Hence $g(\Theta, \Phi)$ belongs to $\mathcal{G}_{\mathfrak{O}}\left(\kappa^{\prime} \psi_{l}, g \mathbf{y}\right)$ for any integer $\kappa^{\prime} \geq n|g| \kappa$. Therefore $g \mathcal{G}_{\mathfrak{O}}^{\prime \prime}(\psi, \mathbf{y})=\mathcal{G}_{\mathfrak{O}}^{\prime \prime}(\psi, g \mathbf{y})$. It follows that if $\mathcal{G}_{\mathfrak{D}}^{\prime \prime}(\psi, \mathbf{y})$ is of full Lebesgue measure for some $\mathbf{y} \neq 0$ then it is of full Lebesgue measure for all $\mathbf{y} \neq 0$. Consequently, the set

$$
\mathcal{G}_{\mathfrak{O}}^{\prime \prime}(\psi):=\left\{(\Theta, \Phi, \mathbf{y}): \mathbf{y} \in \mathbb{R}^{n},(\Theta, \Phi) \in \mathcal{G}_{\mathfrak{O}}^{\prime \prime}(\psi, \mathbf{y})\right\}
$$


is either a full set or a null set in $\operatorname{Mat}_{n, m+n}(\mathbb{R}) \times \mathbb{R}^{n}$. Suppose first that it is a full set. Then in particular there exists $\Phi \in G L(n, \mathbb{R})$ such that its fiber over $\Phi$, namely

$$
F_{\Phi}:=\left\{(\Theta, \mathbf{y}) \in \operatorname{Mat}_{n, m}(\mathbb{R}) \times \mathbb{R}^{n}:(\Theta, \Phi, \mathbf{y}) \in \mathcal{G}_{\mathfrak{O}}^{\prime \prime}(\psi)\right\}
$$

is a full set in $\operatorname{Mat}_{n, m}(\mathbb{R}) \times \mathbb{R}^{n}$. Then $\Phi^{-1} F_{\Phi}=\left\{\left(\Phi^{-1} \Theta, \Phi^{-1} \mathbf{y}\right):(\Theta, \mathbf{y}) \in F_{\Phi}\right\}$ is also a full set. For $\left(\Theta_{1}, \mathbf{y}_{1}\right)=\left(\Phi^{-1} \Theta, \Phi^{-1} \mathbf{y}\right) \in \Phi^{-1} F_{\Phi}$, and any $(\mathbf{q}, \mathbf{p})^{t} \in \mathfrak{O}$ we have

$$
\left|\Theta_{1} \mathbf{q}+\mathbf{p}-\mathbf{y}_{1}\right|=\left|\Phi^{-1}(\Theta \mathbf{q}+\Phi \mathbf{p}-\mathbf{y})\right| \leq n\left|\Phi^{-1}\right||\Theta \mathbf{q}+\Phi \mathbf{p}-\mathbf{y}|
$$

and since $(\Theta, \Phi) \in \mathcal{G}_{\mathfrak{O}}^{\prime \prime}(\psi, \mathbf{y})$ this implies that $\left(\Theta_{1}, \mathbf{y}_{1}\right) \in \mathcal{E}_{\mathfrak{O}}^{\prime}(\psi)$. We have thus shown that $\mathcal{E}_{\mathfrak{D}}^{\prime}(\psi)$ contains the full set $\Phi^{-1} F_{\Phi}$. The inverse argument of multiplication by $\Phi$ show that if $\mathcal{G}_{\mathfrak{O}}^{\prime \prime}(\psi)$ is a null set then $\mathcal{E}_{\mathfrak{O}}^{\prime}(\psi)$ is a null set. This completes the proof.

An analogous (in fact simpler) argument, with $\mathcal{G}_{\mathfrak{O}}^{\prime \prime}(\psi, \mathbf{0})$ playing the role of $\mathcal{G}_{\mathfrak{O}}^{\prime \prime}(\psi)$, shows also the following, for the set as in (2.4), for $\mathbf{y}=\mathbf{0}$; we omit the details.

Proposition 2.3. Let $\Gamma, \mathfrak{O}$ and $\psi$ be as in Proposition 2.1. Then the $\operatorname{set} \mathcal{E}_{\mathfrak{D}}^{\prime}(\psi, \mathbf{0})$ is either a null set or a full set.

Before concluding this section we introduce the subgroups of $S L(m+n, \mathbb{Z})$, and the orbits, to which the above results will be applied in proving the main results. To each partition $\pi$ of $\{1, \ldots, m+n\}$ as $\coprod_{j=1}^{k} \pi_{j}$ we associate the subgroup $\Gamma_{\pi}$ defined as follows. Let $\left\{e_{1}, \ldots, e_{m+n}\right\}$ be the standard basis of $\mathbb{R}^{m+n}$. For each $j$ let $S L\left(\pi_{j}, \mathbb{Z}\right)$ be the subgroup of $S L(m+n, \mathbb{Z})$ consisting of elements that fix $e_{i}$ for all $i \notin \pi_{j}$, and let

$$
\Gamma_{\pi}=\prod_{j=1}^{k} S L\left(\pi_{j}, \mathbb{Z}\right) .
$$

We note that the subgroups $S L\left(\pi_{j}, \mathbb{Z}\right), j=1, \ldots, k$, commute with each other and hence $\Gamma_{\pi}$ is a subgroup of $S L(m+n, \mathbb{Z})$. For any $\pi$ as above, we have

$$
P(\pi)=\Gamma_{\pi}(1, \ldots, 1)^{t},
$$

an orbit of the $\Gamma_{\pi}$-action on $\mathbb{Z}^{m+n}$; these are the orbits $\mathfrak{O}$ involved in the proofs of Theorems 1.1, 1.2 and 1.3, with the partitions $\pi$ as in the theorems. We will be applying the following result on ergodicity, which involves the condition on $\pi$ as in the hypotheses of the theorems.

Proposition 2.4. Let $\pi=\coprod_{j=1}^{k} \pi_{j}$ be a partition of $\{1, \ldots, m+n\}$. Then the action of the group $\Gamma_{\pi}$ on $\mathrm{Mat}_{n, m+n}(\mathbb{R})$, by multiplication on the right, is ergodic if and only if for all $j=1, \ldots, k$ the cardinality of $\pi_{j}$ is at least $n+1$. 
Proof. For each $j$ let $\nu_{j}$ denote the cardinality of $\pi_{j}$. Assume first that $\nu_{j} \geq$ $n+1$ for every $j$ with $1 \leq j \leq k$. Let $\Omega$ be the open subset of $\operatorname{Mat}_{n, m+n}(\mathbb{R})$ consisting of all matrices $M$ such that for each $j$ the $n \times \nu_{j}$ matrix $M_{\pi_{j}}$, formed by columns of $M$ corresponding to the indices in $\pi_{j}$, has rank $n$ (the maximum possible). Clearly $\Gamma_{\pi}$ leaves $\Omega$ invariant. Moreover, the complement of $\Omega$ in $\operatorname{Mat}_{n, m+n}(\mathbb{R})$ is of Lebesgue measure 0 and hence the $\Gamma_{\pi}$-action on $\operatorname{Mat}_{n, m+n}(\mathbb{R})$ is ergodic if and only if the $\Gamma_{\pi}$-action on $\Omega$ is ergodic (with respect to the restricted measure). Via obvious identifications, we can write $\Omega=\prod_{j=1}^{k} \Omega_{n, \nu_{j}}$ where $\Omega_{n, \nu_{j}}$ denotes the open set in $\operatorname{Mat}_{n, \nu_{j}}(\mathbb{R})$ consisting of matrices of rank $n$. Moreover, the action of $\Gamma_{\pi}=\prod_{j=1}^{k} S L\left(\nu_{j}, \mathbb{Z}\right)$ on $\Omega$ is given by componentwise right multiplication. It follows from Moore's ergodicity theorem (see [15], Theorem 5 and Proposition 6; see also [1] ) that the action of the lattice $S L\left(\nu_{j}, \mathbb{Z}\right) \subset S L\left(\nu_{j}, \mathbb{R}\right)$ on $\Omega_{n, \nu_{j}}$ is ergodic, since the latter can be realised as an homogeneous space $\Omega_{n, \nu_{j}}=L_{j} \backslash S L\left(\nu_{j}, \mathbb{R}\right)$ where $L_{j}$ is the stabiliser of a point in $\Omega_{n, \nu_{j}}$, and $L_{j}$ is a non-compact closed subgroup. Thus the action of $\Gamma_{\pi}$ on the product $\Omega$ is as well ergodic.

Suppose now that $\nu_{j} \leq n$ for some $j$. Then the determinant of any $\nu_{j} \times \nu_{j}$ minor extracted from the component $\operatorname{Mat}_{n, \nu_{j}}(\mathbb{R})$ remains invariant under the action of $S L\left(\pi_{j}, \mathbb{R}\right)$ and hence the action is not ergodic.

\section{Generalisation of a lemma of Cassels}

In this section we prove the following metrical proposition which may be viewed as a multi-dimensional analogue of Lemma 3 in [13]. The case of $m=1$, which goes back to Cassels [7], deals with boxes in $\mathbb{R}^{n}$. We are concerned here with a corresponding result for nested infinite sequences of product of strips in $\operatorname{Mat}_{n, m}(\mathbb{R})$, viewed as $\left(\mathbb{R}^{m}\right)^{n}$. Such extensions of Cassels' Lemma occur as well in [2].

Let $\Gamma$ be a subgroup of $S L(m+n, \mathbb{Z})$ and $\mathfrak{O}$ be an infinite orbit of $\Gamma$ in $\mathbb{Z}^{m+n}$. For any function $\psi$, any $\mathbf{y} \in \mathbb{R}^{n}$ and $\Phi \in \operatorname{Mat}_{n, n}(\mathbb{R})$, let

$$
\mathcal{E}_{\mathfrak{O}}(\psi, \Phi, \mathbf{y})=\left\{\Theta \in \operatorname{Mat}_{n, m}(\mathbb{R}):(\Theta, \Phi) \in \mathcal{G}_{\mathfrak{O}}(\psi, \mathbf{y})\right\}
$$

namely, the fiber of $\Phi$ in the set $\mathcal{G}_{\mathfrak{O}}(\psi, \mathbf{y})$ as in $(2.1)$.

Proposition 3.1. Suppose that the function $\ell \mapsto \ell^{-1} \psi(\ell)$ tends to zero as $\ell$ tends to infinity. Then for any $\mathbf{y} \in \mathbb{R}^{n}, \Phi \in G L(n, \mathbb{R})$, and $\kappa \in \mathbb{N}$ the difference

$$
\mathcal{E}_{\mathfrak{O}}(\kappa \psi, \Phi, \mathbf{y}) \backslash \mathcal{E}_{\mathfrak{D}}(\psi, \Phi, \mathbf{y})
$$

is a null set. 
Proof. For $j=1, \ldots, m$ let

$$
\mathfrak{O}_{j}=\left\{(\mathbf{q}, \mathbf{p})^{t} \in \mathfrak{O}:|\mathbf{q}|=\left|q_{j}\right|\right\},
$$

where $q_{j}$ denotes the $j$ th coordinate of $\mathbf{q}$ and for any $\Phi \in G L(n, \mathbb{R})$ let us consider the corresponding set $\mathcal{E}_{\mathfrak{O}_{j}}(\psi, \Phi, \mathbf{y})$. Clearly

$$
\mathcal{E}_{\mathfrak{O}}(\psi, \Phi, \mathbf{y})=\bigcup_{j=1}^{m} \mathcal{E}_{\mathfrak{O}_{j}}(\psi, \Phi, \mathbf{y})
$$

and hence it suffices to show that the difference $\mathcal{E}_{\mathfrak{O}}(\kappa \psi, \Phi, \mathbf{y}) \backslash \mathcal{E}_{\mathfrak{O}}(\psi, \Phi, \mathbf{y})$ is a null set for all $j=1, \ldots, m$. Moreover, by symmetry considerations it suffices to prove this for $j=1$.

We write any $n \times m$ matrix $\Theta \in \operatorname{Mat}_{n, m}(\mathbb{R})$ in the form $\Theta=\left(\xi, \Theta^{\prime}\right)$, where $\xi$ denotes the first column of $\Theta$ and $\Theta^{\prime} \in \operatorname{Mat}_{n, m-1}(\mathbb{R})$ denotes the matrix formed by the remaining columns. For any $\Theta^{\prime} \in \operatorname{Mat}_{n, m-1}(\mathbb{R})$ let

$$
\mathcal{F}\left(\psi, \Theta^{\prime}, \Phi, \mathbf{y}\right)=\left\{\xi \in \mathbb{R}^{n}:\left(\xi, \Theta^{\prime}\right) \in \mathcal{E}_{\mathfrak{O}_{1}}(\psi, \Phi, \mathbf{y})\right\}
$$

be the fiber over $\Theta^{\prime}$ of the subset $\mathcal{E}_{\mathfrak{D}_{1}}(\psi, \Phi, \mathbf{y})$. We write

$$
\Theta^{\prime}=\left(\theta_{1}, \ldots, \theta_{n}\right)^{t} \text { and } \Phi=\left(\phi_{1}, \ldots, \phi_{n}\right)^{t},
$$

where $\theta_{1}, \ldots, \theta_{n} \in \mathbb{R}^{m-1}$ and $\phi_{1}, \ldots, \phi_{n} \in \mathbb{R}^{n}$ are the transposes of the respective rows of $\Theta^{\prime}$ and $\Phi$. For any $\mathbf{v}=(\mathbf{q}, \mathbf{p})^{t} \in \mathfrak{O}_{1}$, with $\mathbf{q}=\left(q_{1}, \mathbf{q}^{\prime}\right)^{t}$ where $q_{1} \in \mathbb{Z} \backslash\{0\}$, $\mathbf{q}^{\prime} \in \mathbb{Z}^{m-1}$, let $\mathcal{F}_{\mathbf{v}}\left(\psi, \Theta^{\prime}, \Phi, \mathbf{y}\right)$ be the set of $\xi \in \mathbb{R}^{n}$ for which the matrices $\left(\left(\xi, \Theta^{\prime}\right), \Phi\right)$ belong to $S_{\mathbf{v}}(\psi, \mathbf{y})$. It is easily seen that

$$
\mathcal{F}_{\mathbf{v}}\left(\psi, \Theta^{\prime}, \Phi, \mathbf{y}\right)=\prod_{i=1}^{n}\left[\frac{-\phi_{i}^{t} \mathbf{p}-\theta_{i}^{t} \mathbf{q}^{\prime}+y_{i}-\psi(|\mathbf{q}|)}{\left|q_{1}\right|}, \frac{-\phi_{i}^{t} \mathbf{p}-\theta_{i}^{t} \mathbf{q}^{\prime}+y_{i}+\psi(|\mathbf{q}|)}{\left|q_{1}\right|}\right]
$$

is an hypercube in $\mathbb{R}^{n}$ and we can write

$$
\mathcal{F}\left(\psi, \Theta^{\prime}, \Phi, \mathbf{y}\right)=\limsup _{\mathbf{v} \in \mathfrak{O}_{1}} \mathcal{F}_{\mathbf{v}}\left(\psi, \Theta^{\prime}, \Phi, \mathbf{y}\right)
$$

as a limsup of hypercubes in $\mathbb{R}^{n}$. Observe that the centers $\left(-\phi_{i}^{t} \mathbf{p}-\theta_{i}^{t} \mathbf{q}^{\prime}+y_{i}\right) /\left|q_{1}\right|$ of the intervals occurring in $\mathcal{F}_{\mathbf{v}}\left(\psi, \Theta^{\prime}, \Phi, \mathbf{y}\right)$ do not depend on $\psi$, and that the length $2 \psi(|\mathbf{q}|) /|\mathbf{q}|$ is multiplied by the constant factor $\kappa$ when $\psi$ is replaced by $\kappa \psi$.

Let $B$ be a compact subset in $\mathbb{R}^{n}$. If for $\mathbf{v}=(\mathbf{q}, \mathbf{p})^{t} \in \mathfrak{O}_{1}$ the intersection $\mathcal{F}_{\mathbf{v}}\left(\kappa \psi, \Theta^{\prime}, \Phi, \mathbf{y}\right) \cap B$ is non-empty, then we have the rough bound $|\mathbf{p}| \leq c \kappa|\mathbf{q}|$ with

$$
c=n\left|\Phi^{-1}\right|\left(|\mathbf{y}|+(m-1)\left|\Theta^{\prime}\right|+\max _{\xi \in B}(|\xi|)+\max _{\ell \geq 1} \frac{\psi(\ell)}{\ell}\right),
$$


and therefore

$$
\mathcal{F}\left(\kappa \psi, \Theta^{\prime}, \Phi, \mathbf{y}\right) \cap B=\limsup _{\mathbf{v} \in \mathfrak{O}_{1},|\mathbf{p}| \leq c \kappa|\mathbf{q}|} \mathcal{F}_{\mathbf{v}}\left(\kappa \psi, \Theta^{\prime}, \Phi, \mathbf{y}\right) \cap B .
$$

Now, fix $\kappa \geq 1$ and set

$$
\mathfrak{O}^{\prime}=\left\{\mathbf{v}=(\mathbf{q}, \mathbf{p})^{t} \in \mathfrak{O}_{1}:|\mathbf{p}| \leq c \kappa|\mathbf{q}|\right\} .
$$

Then we have

$$
\begin{aligned}
\mathcal{F}\left(\psi, \Theta^{\prime}, \Phi, \mathbf{y}\right) \cap B=\limsup _{\mathbf{v} \in \mathfrak{O}^{\prime}} \mathcal{F}_{\mathbf{v}}\left(\psi, \Theta^{\prime}, \Phi, \mathbf{y}\right) \cap B, \text { and } \\
\mathcal{F}\left(\kappa \psi, \Theta^{\prime}, \Phi, \mathbf{y}\right) \cap B=\limsup _{\mathbf{v} \in \mathfrak{O}^{\prime}} \mathcal{F}_{\mathbf{v}}\left(\kappa \psi, \Theta^{\prime}, \Phi, \mathbf{y}\right) \cap B
\end{aligned}
$$

If $\mathfrak{D}^{\prime}$ is finite, both the sets $\mathcal{F}\left(\psi, \Theta^{\prime}, \Phi, \mathbf{y}\right) \cap B$ and $\mathcal{F}\left(\kappa \psi, \Theta^{\prime}, \Phi, \mathbf{y}\right) \cap B$ are empty. If the set $\mathfrak{O}^{\prime}$ is infinite, we enumerate it as a sequence $\left\{\mathbf{v}_{h}=\left(\mathbf{q}_{h}, \mathbf{p}_{h}\right)^{t}\right\}_{h=1}^{\infty}$. Then, $\left|\mathbf{q}_{h}\right|$ tends to infinity as $h$ tends to infinity, since there are only finitely many $(\mathbf{q}, \mathbf{p})^{t} \in \mathfrak{O}^{\prime}$ with $|\mathbf{q}|$ bounded. Therefore the length $2 \kappa \psi\left(\left|\mathbf{q}_{h}\right|\right) /\left|\mathbf{q}_{h}\right|$ of the sides of the hypercube $\mathcal{F}_{\mathbf{v}_{h}}\left(\kappa \psi, \Theta^{\prime}, \Phi, \mathbf{y}\right)$ tends to zero as $h$ tends infinity, since we have assumed that $\lim _{\ell \mapsto+\infty} \psi(\ell) / \ell=0$. We may thus apply the classical lemma of Cassels, namely Lemma 9 in [7] (see also Lemma 2.1 in [10]), to the nested sequences of hypercubes

$$
\mathcal{F}_{\mathbf{v}_{h}}\left(\psi, \Theta^{\prime}, \Phi, \mathbf{y}\right) \subseteq \mathcal{F}_{\mathbf{v}_{h}}\left(\kappa \psi, \Theta^{\prime}, \Phi, \mathbf{y}\right), \text { for all } h \geq 1
$$

The Lemma asserts that the associated upper limit sets

$$
\mathcal{F}\left(\psi, \Theta^{\prime}, \Phi, \mathbf{y}\right) \cap B \subseteq \mathcal{F}\left(\kappa \psi, \Theta^{\prime}, \Phi, \mathbf{y}\right) \cap B
$$

have the same Lebesgue measure. Since this holds for any compact set $B$ it follows that all the sets $\mathcal{F}\left(\kappa \psi, \Theta^{\prime}, \Phi, \mathbf{y}\right), \kappa \geq 1$, are equal to each other up to a null set. Applying Fubini theorem we infer that the fibered sets $\mathcal{E}_{\mathfrak{O}}(\kappa \psi, \Phi, \mathbf{y}), \kappa \geq 1$, coincide up to null sets.

The special case $\Phi=I_{n}$ of Proposition 3.1 leads to the following result for the sets $\mathcal{E}_{\mathfrak{O}}(\psi, \mathbf{y})$ as in $(2.2)$.

Corollary 3.2. Let $\psi$ be as in Proposition [3.1. Then for any $\mathbf{y} \in \mathbb{R}^{n}, \kappa \in \mathbb{N}$ the difference $\mathcal{E}_{\mathfrak{D}}(\kappa \psi, \mathbf{y}) \backslash \mathcal{E}_{\mathfrak{O}}(\psi, \mathbf{y})$ is a set of Lebesgue measure 0.

\section{Some arithmetical estimates}

In this section we digress and collect some elementary counting results for use in the estimates needed in the next section. 
Lemma 4.1. Let $\beta>0$ and $0<\epsilon<1$ be given. Then for all pairs of positive integers $q$ and $Q$ such that $q \leq Q$, with $Q$ large enough, the number of the integers $n$ satisfying

$$
1 \leq n \leq \beta Q \quad \text { and } \quad \operatorname{gcd}(n, q)=1
$$

is at least

$$
(1-\epsilon) \beta Q \prod_{\substack{p \mid q \\ p \text { prime }}}\left(1-\frac{1}{p}\right)=(1-\epsilon) \beta Q \frac{\varphi(q)}{q},
$$

where $\varphi$ denotes the Euler totient function.

Proof. By the sieving formula of Legendre-Eratosthenes, the number $N$ of these integers $n$ located in the interval $[1, \beta Q]$ and coprime with $q$ is given by the expression

$$
N=\sum_{d \mid q} \mu(d)\left[\frac{\beta Q}{d}\right]
$$

where $\mu$ stands as usual for the Möbius function. Recall that $\mu(d)$ vanishes unless $d$ is square-free. Therefore

$$
\begin{aligned}
N & =\beta Q\left(\sum_{d \mid q} \frac{\mu(d)}{d}\right)-\sum_{d \mid q} \mu(d)\left\{\frac{\beta Q}{q}\right\} \\
& =\beta Q \prod_{\substack{p \mid q \\
p \text { prime }}}\left(1-\frac{1}{p}\right)+O\left(2^{\omega(q)}\right),
\end{aligned}
$$

where $\omega(q)$ denotes the number of prime divisors of $q$. Now, it is well-known that

$$
\prod_{\substack{p \mid q \\ p \text { prime }}}\left(1-\frac{1}{p}\right)=\frac{\varphi(q)}{q} \geq\left(e^{-\gamma}-o(1)\right) \frac{1}{\log \log q} \geq\left(e^{-\gamma}-o(1)\right) \frac{1}{\log \log Q},
$$

where $\gamma$ is the Euler constant, while

$$
\omega(q) \leq(1+o(1)) \frac{\log q}{\log \log q} \leq(1+o(1)) \frac{\log Q}{\log \log Q}
$$

Thus, $N$ is asymptotically equivalent to the first term $\beta Q \prod_{\substack{p \mid q \\ p \text { prime }}}\left(1-\frac{1}{p}\right)$ as $Q$ tends to infinity, uniformly for $q$ ranging along the interval $1 \leq q \leq Q$. 
Lemma 4.2. Let $d$ be an integer $\geq 2$ and $Q$ be a positive real number. Then the number of points

$$
\mathbf{q} \in P\left(\mathbb{Z}^{d}\right) \cap \mathbb{N}^{d} \quad \text { with } \quad|\mathbf{q}| \leq Q
$$

is asymptotically equivalent to $\zeta(d)^{-1} Q^{d}$ as $Q$ tends to infinity.

Proof. The number of points $\mathbf{q}$ in $P\left(\mathbb{Z}^{d}\right)$ with norm $|\mathbf{q}| \leq Q$ is asymptotically equivalent to $2^{d} \zeta(d)^{-1} Q^{d}$ as $Q$ tends to infinity; this assertion may be deduced from the special case $K=\mathbb{Q}$ of Schanuel's Theorem estimating the number of points in $\mathbb{P}^{d-1}(K)$ with height bounded by $Q$ for any fixed number field $K$ (see for instance Theorem 5.3 in [12]). Now, each of the $2^{d}$ possible quadrants have the same number of primitive points contained in the box $|\mathbf{q}| \leq Q$.

Putting together the two results we next obtain an estimate on certain sets of primitive points which will be used in the next section in estimating measures of certain sets.

We first note that given a partition $\pi=\coprod_{j=1}^{k} \pi_{j}$ of $\{1, \ldots, m+n\}$, where $m, n \in$ $\mathbb{N}$, by renumbering the indices suitably we can arrange so that the following holds: there exist indices $a$ and $b$ with $0 \leq a \leq \min (k, m, n)$ and $a \leq b \leq k$ such that $\{j, m+j\} \subset \pi_{j}$ for $1 \leq j \leq a, \pi_{j} \subset\{m+1, \ldots, m+n\}$ for $a+1 \leq j \leq b$, and $\pi_{j} \subset\{1, \ldots, m\}$ for $b+1 \leq j \leq k$. In other words, the components $\pi_{1}, \ldots, \pi_{a}$ are those whose intersection with both intervals $\{1, \ldots, m\}$ and $\{m+1, \ldots, m+n\}$ is non-empty, while the other components are contained either in $\{1, \ldots, m\}$ or in $\{m+1, \ldots, m+n\}$. The case $a=0$ means that there is no component of the first type.

Corollary 4.3. Let $m, n \in \mathbb{N}$ and let $\pi=\coprod_{j=1}^{k} \pi_{j}$ be a partition of $\{1, \ldots, m+n\}$ such that each $\pi_{j}$ has at least 2 elements. Suppose that the condition as above holds, with $0 \leq a \leq b \leq k$. For $a+1 \leq j \leq b$ let $d_{j}$ denote the cardinality of $\pi_{j}$. Let $\beta>0$ and $\epsilon>0$ be given. Then for any $\mathbf{q}=\left(q_{1}, \ldots, q_{m}\right)^{t} \in \mathbb{N}^{m}$ such that $\mathbf{q} \in P\left(\pi_{j}\right)$ for all $j \geq b+1$, and $|\mathbf{q}|$ is sufficiently large we have

$$
\operatorname{card}\left\{\mathbf{p} \in \mathbb{N}^{\mathrm{n}}:(\mathbf{q}, \mathbf{p})^{\mathrm{t}} \in \mathrm{P}(\pi),|\mathbf{p}| \leq \beta|\mathbf{q}|\right\} \geq(1-\epsilon) \beta^{\mathrm{n}}|\mathbf{q}|^{\mathrm{n}} \prod_{\mathrm{j}=\mathrm{a}+1}^{\mathrm{b}} \zeta\left(\mathrm{d}_{\mathrm{j}}\right)^{-1} \prod_{\mathrm{j}=1}^{\mathrm{a}} \frac{\varphi\left(\mathrm{q}_{\mathrm{j}}\right)}{\mathrm{q}_{\mathrm{j}}}
$$

Proof. For $a+1 \leq j \leq b$ and $\mathbf{p} \in \mathbb{N}^{n}$ let $\mathbf{p}_{j}$ denote the projection of $\mathbf{p}$ to the coordinates corresponding to $\pi_{j}$ (the latter is contained in $\{m+1, \ldots, m+n\}$ ). Also let $d=\sum_{j=a+1}^{b} d_{j}$. Consider the set, say $P^{\prime}$, of $\mathbf{p}=\left(p_{1}, \ldots, p_{n}\right)^{t} \in \mathbb{N}^{n}$ such that (i) $p_{j} \leq \beta|\mathbf{q}|$ and $\operatorname{gcd}\left(q_{j}, p_{j}\right)=1$ for $1 \leq j \leq a$, (ii) $\left|\mathbf{p}_{j}\right| \leq \beta|\mathbf{q}|$ and $\mathbf{p} \in P\left(\pi_{j}\right)$ for $a+1 \leq j \leq b$, and (iii) $|\mathbf{p}| \leq \beta|\mathbf{q}|$ (the last part applies afresh only to the remaining $n-a-d$ coordinates of $\mathbf{p}$ not covered in (i) and (ii)). We note that $P^{\prime}$ is contained in 
the set on the left hand side in the inequality as above, and the desired cardinality is at least $\operatorname{card}\left(\mathrm{P}^{\prime}\right)$. On the other hand to pick an element of $P^{\prime}$ the choices for $p_{1}, \ldots, p_{a}, \mathbf{p}_{j}, a+1 \leq j \leq b$, and the remaining $n-a-d$ coordinates may be made independently, satisfying the respective conditions (i), (ii) and (iii). Therefore when $\mathbf{q}$ is sufficiently large by Lemmas 4.1 and 4.2 we get that card $\left(\mathrm{P}^{\prime}\right)$ is bounded below by the product of $(1-\epsilon / 3) \beta^{a}|\mathbf{q}|^{a} \prod_{j=1}^{a} \frac{\varphi\left(q_{j}\right)}{q_{j}},(1-\epsilon / 3) \beta^{d}|\mathbf{q}|^{d} \prod_{j=a+1}^{b} \zeta\left(d_{j}\right)^{-1}$ and $(1-\epsilon / 3) \beta^{n-a-d}|\mathbf{q}|^{n-a-d}$, corresponding to the choices to be made. This shows that the estimate as stated in the corollary holds.

\section{$5 \quad$ Estimating measures of sets}

Our next objective will be to obtain an estimate as in the following theorem, which together with the results of the earlier sections will enable us complete the proofs of Theorems 1.1, 1.2 and 1.3. Though the components of the partition involved in them are assumed to have at least $n+1$ elements, for Theorem 5.1 we need only the weaker condition as in Corollary 4.3, the general form may turn out to be of independent interest.

Let

$$
\mathbb{I}=\left\{\Theta \in \operatorname{Mat}_{n, m}(\mathbb{R}):|\Theta| \leq \frac{1}{2}\right\}
$$

(as before $|\cdot|$ stands for the supremum norm).

Theorem 5.1. Let $m, n \in \mathbb{N}$, not both equal to 1 , and let $\pi$ be a partition of $\{1, \ldots, m+n\}$ such that each component of $\pi$ has at least 2 elements. Let $\lambda$ be the Lebesgue measure on $\mathrm{Mat}_{n, m}(\mathbb{R})$. Then there exists a constant $\delta>0$ such that the following holds: for any function $\psi: \mathbb{N} \rightarrow\left(0, \frac{1}{2}\right)$ such that the mapping $x \mapsto x^{m-1} \psi(x)^{n}$ is non-increasing and

$$
\sum_{j \geq 1} j^{m-1} \psi(j)^{n}=\infty
$$

and any $\mathbf{y} \in \mathbb{R}^{n}$ we have

$$
\lambda\left(\mathcal{E}_{P(\pi)}(\psi, \mathbf{y}) \cap \mathbb{I}\right) \geq \delta
$$

The proof of this theorem will be completed in the next section. In this section we first establish various estimates needed in the proof. For simplicity of notation, through the proof we shall suppress $\psi$ from the notation for various sets defined along the way. We note that the constant $\delta$ is meant to be chosen independently of $\psi$, which will be ensured separately in the course of the argument. 
We introduce for any $\mathbf{y} \in \mathbb{R}^{n}$ and any $\mathbf{v}=(\mathbf{q}, \mathbf{p})^{t} \in \mathbb{Z}^{m+n}$ the 'strip'

$$
R_{\mathbf{v}}(\mathbf{y}):=\left\{\Theta \in \operatorname{Mat}_{n, m}(\mathbb{R}) ; \quad|\Theta \mathbf{q}+\mathbf{p}-\mathbf{y}| \leq \psi(|\mathbf{q}|)\right\}
$$

For any $\mathbf{q} \in \mathbb{Z}^{m}$, let

$$
\Lambda(\mathbf{q})=\left\{\mathbf{v} \in P(\pi): \mathbf{v}=(\mathbf{q}, \mathbf{p})^{t} \text { for some } \mathbf{p} \in \mathbb{Z}^{n}\right\}
$$

(which could possibly be empty) and

$$
E_{\mathbf{q}}(\mathbf{y})=\bigcup_{\mathbf{v} \in \Lambda(\mathbf{q})} R_{\mathbf{v}}(\mathbf{y})
$$

Since $\psi(|\mathbf{q}|)<\frac{1}{2}$, this is a union of disjoint sets. The fiber $\mathcal{E}_{P(\pi)}(\psi, \mathbf{y})$ of $\mathcal{E}_{P(\pi)}(\psi)$ over $\mathbf{y} \in \mathbb{R}^{n}$ is then equal to the limsup set

$$
\mathcal{E}_{P(\pi)}(\psi, \mathbf{y})=\bigcap_{Q \geq 1|\mathbf{q}| \geq Q} \bigcup_{\mathbf{q}} E_{\mathbf{y}}(\mathbf{y}
$$

As usual when dealing with limsup set in metrical theory, we first estimate the Lebesgue measure of pairwise intersections of the subsets $E_{\mathbf{q}}(\mathbf{y}), \mathbf{q} \in \mathbb{Z}^{m}$. We begin by upper bounds. See [3, 4, 6, 8, 10, 16, 17, 18, 19, 21] for various 'overlapping' estimates of this kind which are a keystone in metrical diophantine approximation.

Lemma 5.2. The following estimates hold for any $\mathbf{y} \in \mathbb{R}^{n}$ :

(i) For any non-zero $\mathbf{q} \in \mathbb{Z}^{m}$,

$$
\lambda\left(E_{\mathbf{q}}(\mathbf{y}) \cap \mathbb{I}\right) \leq 2^{n} \psi(|\mathbf{q}|)^{n} .
$$

(ii) For any linearly independent vectors $\mathbf{q}$ and $\mathbf{q}^{\prime}$ in $\mathbb{Z}^{m}$,

$$
\lambda\left(E_{\mathbf{q}}(\mathbf{y}) \cap E_{\mathbf{q}^{\prime}}(\mathbf{y}) \cap \mathbb{I}\right) \leq 4^{n} \psi(|\mathbf{q}|)^{n} \psi\left(\left|\mathbf{q}^{\prime}\right|\right)^{n} .
$$

(iii) For $\mathbf{q}=q \mathbf{a}$ and $\mathbf{q}^{\prime}=q^{\prime} \mathbf{a}$, where $q$ and $q^{\prime}$ are coprime integers with $|q| \geq$ $\left|q^{\prime}\right|>0$ and $\mathbf{a}$ is a non-zero vector in $\mathbb{Z}^{m}$, we have

$$
\lambda\left(E_{\mathbf{q}}(\mathbf{y}) \cap E_{\mathbf{q}^{\prime}}(\mathbf{y}) \cap \mathbb{I}\right) \leq 12^{n} \psi(|\mathbf{q}|)^{n} \max \left(\psi\left(\left|\mathbf{q}^{\prime}\right|\right)^{n},|q|^{-n}\right) .
$$

Proof. Since we are concerned with upper bounds, it will be convenient to deal in place of $E_{\mathbf{q}}(\mathbf{y})$ with the larger set

$$
F_{\mathbf{q}}(\mathbf{y}):=\bigcup_{\mathbf{p} \in \mathbb{Z}^{n}} R_{\mathbf{q}, \mathbf{p})^{t}}(\mathbf{y})=\left\{\Theta \in \operatorname{Mat}_{n, m}(\mathbb{R}) ;\|\Theta \mathbf{q}-\mathbf{y}\| \leq \psi(|\mathbf{q}|)\right\}
$$


where $\|\cdot\|$ stands, as usual, for the distance to the nearest point of $\mathbb{Z}^{n}$. Let us denote by $\mathbb{T}^{n}$ the $n$-dimensional torus $(\mathbb{R} / \mathbb{Z})^{n}$ and let $\eta: \mathbb{R}^{n} \rightarrow \mathbb{T}^{n}$ be the canonical quotient map. With some obvious abuse of notation, we introduce for any $\mathbf{y} \in \mathbb{R}^{n}$, $q \in \mathbb{Z} \backslash\{0\}$ and $r>0$ the two subsets

$$
A_{q}(\mathbf{y}, r)=\left\{\mathbf{z} \in \mathbb{R}^{n}:|q \mathbf{z}-\mathbf{y}| \leq r\right\} \quad \text { and } \quad B_{q}(\mathbf{y}, r)=\left\{\mathbf{z} \in \mathbb{T}^{n}:\|q \mathbf{z}-\mathbf{y}\| \leq r\right\} .
$$

Thus

$$
B_{q}(\mathbf{y}, r)=\bigcup_{\mathbf{p} \in \mathbb{Z}^{n}} \eta\left(A_{q}(\mathbf{y}-\mathbf{p}, r)\right)=\bigcup_{\mathbf{p} \in \mathbb{Z}^{n} / q \mathbb{Z}^{n}} \eta\left(A_{q}(\mathbf{y}-\mathbf{p}, r)\right) .
$$

noting that we can restrict the index $\mathbf{p}$ in the first union to range along a complete set of representatives modulo $q$, since $\eta\left(A_{q}(\mathbf{y}-\mathbf{p}, r)\right)=\eta\left(A_{q}\left(\mathbf{y}-\mathbf{p}_{1}, r\right)\right)$ when $\mathbf{p} \equiv \mathbf{p}_{1} \bmod q \mathbb{Z}^{n}$. For any $\mathbf{q} \in \mathbb{Z}^{m} \backslash\{0\}$, we introduce the map $T_{\mathbf{q}}: \mathbb{I} \rightarrow \mathbb{T}^{n}$ defined by $T_{\mathbf{q}}(\Theta)=\eta(\Theta \mathbf{q})$ for all $\Theta \in \mathbb{I}$, so that

$$
F_{\mathbf{q}}(\mathbf{y}) \cap \mathbb{I}=T_{\mathbf{q}}^{-1}\left(B_{1}(\mathbf{y}, \psi(|\mathbf{q}|)) .\right.
$$

i) We equip the torus $\mathbb{T}^{n}$ with the Haar measure $\omega$ normalized by $\omega\left(\mathbb{T}^{n}\right)=1$ and the hypercube $\mathbb{I}$ with the Lebesgue measure $\lambda$. By a formula of Sprindzuck (see [19], formula (48) on page 35) $T_{\mathbf{q}}$ is measure-preserving, and hence

$$
\lambda\left(F_{\mathbf{q}}(\mathbf{y}) \cap \mathbb{I}\right)=\omega\left(B_{1}(\mathbf{y}, \psi(|\mathbf{q}|))=2^{n} \psi(|\mathbf{q}|)^{n},\right.
$$

which proves assertion (i).

ii) Let $\mathbf{y} \in \mathbb{R}^{n}$ and $\mathbf{q}$ and $\mathbf{q}^{\prime}$ be linearly independent vectors in $\mathbb{Z}^{m}$. Consider the two maps

$$
T_{\mathbf{q}}: \mathbb{I} \mapsto \mathbb{T}^{n} \quad \text { and } \quad T_{\mathbf{q}^{\prime}}: \mathbb{I} \mapsto \mathbb{T}^{n},
$$

sending $\Theta \in \mathbb{I}$ to $\eta(\Theta \mathbf{q})$ and $\eta\left(\Theta \mathbf{q}^{\prime}\right)$ respectively. Then, the product mapping $T_{\mathbf{q}} \times T_{\mathbf{q}^{\prime}}: \mathbb{I} \mapsto \mathbb{T}^{n} \times \mathbb{T}^{n}$ is as well measure-preserving, where $\mathbb{T}^{n} \times \mathbb{T}^{n}$ is equipped with the product measure $\omega \times \omega$; this may be seen from formula (49) in [19]. Since

$$
F_{\mathbf{q}}(\mathbf{y}) \cap F_{\mathbf{q}^{\prime}}(\mathbf{y}) \cap \mathbb{I}=\left(T_{\mathbf{q}} \times T_{\mathbf{q}^{\prime}}\right)^{-1}\left(B_{1}(\mathbf{y}, \psi(|\mathbf{q}|)) \times B_{1}\left(\mathbf{y}, \psi\left(\left|\mathbf{q}^{\prime}\right|\right)\right)\right),
$$

we obtain the equality

$\lambda\left(F_{\mathbf{q}}(\mathbf{y}) \cap F_{\mathbf{q}^{\prime}}(\mathbf{y}) \cap \mathbb{I}\right)=\omega \times \omega\left(B_{1}(\mathbf{y}, \psi(|\mathbf{q}|)) \times B_{1}\left(\mathbf{y}, \psi\left(\left|\mathbf{q}^{\prime}\right|\right)\right)\right)=4^{n} \psi(|\mathbf{q}|)^{n} \psi\left(\left|\mathbf{q}^{\prime}\right|\right)^{n}$

which yields (ii). 
iii) Let $\mathbf{y} \in \mathbb{R}^{n}, \mathbf{a} \in \mathbb{Z}^{m} \backslash\{0\}, q, q^{\prime} \in \mathbb{Z}$ be two coprime integers and let $\mathbf{q}=q \mathbf{a}$ and $\mathbf{q}^{\prime}=q^{\prime} \mathbf{a}$. Then

$$
F_{\mathbf{q}}(\mathbf{y}) \cap F_{\mathbf{q}^{\prime}}(\mathbf{y}) \cap \mathbb{I}=T_{\mathbf{a}}^{-1}\left(B_{q}(\mathbf{y}, \psi(|\mathbf{q}|)) \cap B_{q^{\prime}}\left(\mathbf{y}, \psi\left(\left|\mathbf{q}^{\prime}\right|\right)\right)\right),
$$

Since $T_{\mathbf{a}}$ is measure-preserving, we have

$$
\lambda\left(F_{\mathbf{q}}(\mathbf{y}) \cap F_{\mathbf{q}^{\prime}}(\mathbf{y}) \cap \mathbb{I}\right)=\omega\left(B_{q}(\mathbf{y}, \psi(|\mathbf{q}|)) \cap B_{q^{\prime}}\left(\mathbf{y}, \psi\left(\left|\mathbf{q}^{\prime}\right|\right)\right)\right) .
$$

We claim that

$$
\omega\left(B_{q}(\mathbf{y}, \psi(|\mathbf{q}|)) \cap B_{q^{\prime}}\left(\mathbf{y}, \psi\left(\left|\mathbf{q}^{\prime}\right|\right)\right)\right) \leq 12^{n} \psi(|\mathbf{q}|)^{n} \max \left(\psi\left(\left|\mathbf{q}^{\prime}\right|\right), \frac{1}{|q|}\right)^{n},
$$

which will yield (iii). The set $B_{q}(\mathbf{y}, \psi(|\mathbf{q}|)) \cap B_{q^{\prime}}\left(\mathbf{y}, \psi\left(\left|\mathbf{q}^{\prime}\right|\right)\right)$ is the image under $\eta$ of its inverse image

$$
\bigcup_{\mathbf{p} \in \mathbb{Z}^{n}} \bigcup_{\mathbf{p}^{\prime} \in \mathbb{Z}^{n}}\left(A_{q}(\mathbf{y}-\mathbf{p}, \psi(|\mathbf{q}|)) \cap A_{q^{\prime}}\left(\mathbf{y}-\mathbf{p}^{\prime}, \psi\left(\left|\mathbf{q}^{\prime}\right|\right)\right)\right) .
$$

For $\mathbf{p}, \mathbf{p}^{\prime} \in \mathbb{Z}^{n}$ let us set $\alpha\left(\mathbf{p}, \mathbf{p}^{\prime}\right):=q \mathbf{p}^{\prime}-q^{\prime} \mathbf{p}$. The intersection

$$
A_{q}(\mathbf{y}-\mathbf{p}, \psi(|\mathbf{q}|)) \cap A_{q^{\prime}}\left(\mathbf{y}-\mathbf{p}^{\prime}, \psi\left(\left|\mathbf{q}^{\prime}\right|\right)\right)
$$

indexed by the pair $\left(\mathbf{p}, \mathbf{p}^{\prime}\right)$ is non-empty only when the distance between $q^{-1}(\mathbf{y}-\mathbf{p})$ and $q^{\prime-1}\left(\mathbf{y}-\mathbf{p}^{\prime}\right)$ is at most $|q|^{-1} \psi(|\mathbf{q}|)+\left|q^{\prime}\right|^{-1} \psi\left(\left|\mathbf{q}^{\prime}\right|\right)$, or equivalently when

$$
\left|\alpha\left(\mathbf{p}, \mathbf{p}^{\prime}\right)-\left(q-q^{\prime}\right) \mathbf{y}\right| \leq\left|q^{\prime}\right| \psi(|\mathbf{q}|)+|q| \psi\left(\left|\mathbf{q}^{\prime}\right|\right) .
$$

Observe that for two integer pairs $\left(\mathbf{p}, \mathbf{p}^{\prime}\right)$ and $\left(\mathbf{p}_{1}, \mathbf{p}_{1}^{\prime}\right)$, we have $\alpha\left(\mathbf{p}, \mathbf{p}^{\prime}\right)=\alpha\left(\mathbf{p}_{1}, \mathbf{p}_{1}^{\prime}\right)$ if and only if $\mathbf{p}_{1}=\mathbf{p}+q \mathbf{r}$ and $\mathbf{p}_{1}^{\prime}=\mathbf{p}^{\prime}+q^{\prime} \mathbf{r}$ for some $\mathbf{r} \in \mathbb{Z}^{n}$, since $q$ and $q^{\prime}$ are coprime integers. Then

$$
A_{q}\left(\mathbf{y}-\mathbf{p}_{1}, \psi(|\mathbf{q}|)\right)=A_{q}(\mathbf{y}-\mathbf{p}, \psi(|\mathbf{q}|))-\mathbf{r}, \quad A_{q^{\prime}}\left(\mathbf{y}-\mathbf{p}_{1}^{\prime}, \psi\left(\left|\mathbf{q}^{\prime}\right|\right)\right)=A_{q^{\prime}}\left(\mathbf{y}-\mathbf{p}^{\prime}, \psi\left(\left|\mathbf{q}^{\prime}\right|\right)\right)-\mathbf{r},
$$

so that the associated intersections $A_{q}\left(\mathbf{y}-\mathbf{p}_{1}, \psi(|\mathbf{q}|)\right) \cap A_{q^{\prime}}\left(\mathbf{y}-\mathbf{p}_{1}^{\prime}, \psi\left(\left|\mathbf{q}^{\prime}\right|\right)\right)$ and $A_{q}(\mathbf{y}-\mathbf{p}, \psi(|\mathbf{q}|)) \cap A_{q^{\prime}}\left(\mathbf{y}-\mathbf{p}^{\prime}, \psi\left(\left|\mathbf{q}^{\prime}\right|\right)\right)$ have the same image under $\eta$. Hence, for each $\mathbf{z} \in \mathbb{Z}^{n}$ satisfying

$$
\left|\mathbf{z}-\left(q-q^{\prime}\right) \mathbf{y}\right| \leq\left|q^{\prime}\right| \psi(|\mathbf{q}|)+|q| \psi\left(\left|\mathbf{q}^{\prime}\right|\right),
$$

it suffices to keep only one pair $\left(\mathbf{p}, \mathbf{p}^{\prime}\right)$ with $\alpha\left(\mathbf{p}, \mathbf{p}^{\prime}\right)=\mathbf{z}$ included in the union as above. The number of these integer points $\mathbf{z}$ is at most $\left(3 \max \left(\left|q^{\prime}\right| \psi(|\mathbf{q}|)+|q| \psi\left(\left|\mathbf{q}^{\prime}\right|\right), 1\right)\right)^{n}$ and since $\left|q^{\prime}\right| \leq|q|$ it is majorised by

$$
\left(3 \max \left(2|q| \psi\left(\left|\mathbf{q}^{\prime}\right|\right), 1\right)\right)^{n} \leq 6^{n}|q|^{n} \max \left(\psi\left(\left|\mathbf{q}^{\prime}\right|\right)^{n},|q|^{-n}\right) .
$$


Also if $\mathbf{p}, \mathbf{p}^{\prime}$ is such that $\alpha\left(\mathbf{p}, \mathbf{p}^{\prime}\right)=\mathbf{z}$, then the Lebesgue measure of the corresponding intersection $A_{q}(\mathbf{y}-\mathbf{p}, \psi(|\mathbf{q}|)) \cap A_{q^{\prime}}\left(\mathbf{y}-\mathbf{p}^{\prime}, \psi\left(\left|\mathbf{q}^{\prime}\right|\right)\right)$ is obviously bounded by

$$
\lambda\left(A_{q}(\mathbf{y}-\mathbf{p}, \psi(|\mathbf{q}|))=2^{n}|q|^{-n} \psi(|\mathbf{q}|)^{n} .\right.
$$

Together with the preceding observation this proves the claim in (5.2) and hence also (iii).

In the opposite direction, we now obtain a lower bound on an average.

Lemma 5.3. Let $m, n$ and $\pi$ be as in the statement of Theorem 5.1. There exists a positive real number $c$, such that for any function $\psi$ satisfying the conditions as in hypothesis of Theorem 5.1, any $\mathbf{y} \in \mathbb{R}^{n}$ and any sufficiently large integer $Q$, we have the lower bound

$$
\sum_{\mathbf{q} \in \mathbb{Z}^{m}, 1 \leq|\mathbf{q}| \leq Q} \lambda\left(E_{\mathbf{q}}(\mathbf{y}) \cap \mathbb{I}\right) \geq c \sum_{\ell=1}^{Q} \ell^{m-1} \psi(\ell)^{n} .
$$

Proof. In the sequel, we indicate by $c_{1}, \ldots$ positive real numbers which, as well as $c$, depend only on $m$ and $n$ (prima facie they may depend on $\pi$, but for the latter there are only finitely many possibilities).

Denote by $|\mathbf{q}|_{2}$ the euclidean norm of the $m$-tuple $\mathbf{q}$ and observe that $|\mathbf{q}| \leq|\mathbf{q}|_{2}$. We claim that for all $\mathbf{v}=(\mathbf{q}, \mathbf{p})^{t}$ with $\mathbf{q} \in \mathbb{Z}^{m} \backslash\{0\}, \mathbf{p} \in \mathbb{Z}^{n}$ such that $|\mathbf{p}| \leq \frac{1}{6}|\mathbf{q}|_{2}$ we have

$$
\lambda\left(R_{\mathbf{v}}(\mathbf{y}) \cap \mathbb{I}\right) \geq\left(\frac{\psi(|\mathbf{q}|)}{2^{m-2}(m-1) !|\mathbf{q}|_{2}}\right)^{n},
$$

when $|\mathbf{q}|$ is sufficiently large. Write the inequality $|\Theta \mathbf{q}+\mathbf{p}-\mathbf{y}| \leq \psi(|\mathbf{q}|)$ in the equivalent form

$$
\left|\xi_{i} \cdot \mathbf{u}-v_{i}\right| \leq|\mathbf{q}|_{2}^{-1} \psi(|\mathbf{q}|), \quad 1 \leq i \leq n
$$

where $\xi_{1}, \ldots, \xi_{n}$ are the (column) vectors in $\mathbb{R}^{m}$ which are the transposes of the $n$ rows of $\Theta$ and

$$
\mathbf{u}=\frac{\mathbf{q}}{|\mathbf{q}|_{2}}, \quad v_{i}=\frac{y_{i}-p_{i}}{|\mathbf{q}|_{2}} \quad \text { for } \quad 1 \leq i \leq n .
$$

(the dot stands for the usual scalar product in $\mathbb{R}^{m}$ ). Note that $|\mathbf{u}|_{2}=1$. Since $|\mathbf{p}| \leq \frac{1}{6}|\mathbf{q}|_{2}$, we have

$$
\max _{1 \leq i \leq n}\left|v_{i}\right| \leq \frac{1}{5}
$$


when $|\mathbf{q}|$ is large enough. Slicing the euclidean ball $\left\{\eta \in \mathbb{R}^{m}:|\eta|_{2} \leq \frac{1}{2}\right\}$ by the hyperplanes $\eta \cdot \mathbf{u}=v_{i}, 1 \leq i \leq n$, we see that the set of points $\xi_{i}$ in $\mathbb{R}^{m}$ satisfying (5.4) and contained in the hypercube $|\eta| \leq \frac{1}{2}$ has Lebesgue measure at least

$$
\sigma_{m-1}\left(\frac{1}{4}\right)^{m-1} \frac{2 \psi(|\mathbf{q}|)}{|\mathbf{q}|_{2}} \geq \frac{\psi(|\mathbf{q}|)}{2^{m-2}(m-1) !|\mathbf{q}|_{2}}
$$

where $\sigma_{m-1}$ denotes the volume of the unit euclidean ball in $\mathbb{R}^{m-1}$. Since $R_{\mathbf{v}}(\mathbf{y})$ is a product set in $\left(\mathbb{R}^{m}\right)^{n}$, this establishes (5.3).

For any $\mathbf{q} \in \mathbb{Z}^{m} \backslash\{0\}$, denote by $N_{\pi}(\mathbf{q})$ the number of $\mathbf{p} \in \mathbb{Z}^{n}$ such that

$$
(\mathbf{q}, \mathbf{p})^{t} \in P(\pi) \quad \text { and } \quad|\mathbf{p}| \leq \frac{1}{6}|\mathbf{q}| \text {. }
$$

It follows from (5.3), with the notation $\Lambda(\mathbf{q})$ as before (see (5.1)), that

$$
\lambda\left(E_{\mathbf{q}}(\mathbf{y}) \cap \mathbb{I}\right)=\sum_{\mathbf{v} \in \Lambda(\mathbf{q})} \lambda\left(R_{\mathbf{v}}(\mathbf{y}) \cap \mathbb{I}\right) \geq N_{\pi}(\mathbf{q})\left(\frac{\psi(|\mathbf{q}|)}{2^{m-2}(m-1) !|\mathbf{q}|_{2}}\right)^{n} .
$$

We shall now use the estimate provided by Corollary 4.3. Renumbering the indices if necessary, as in the comment just before the statement of the corollary, we assume that $\pi$ is expressed as $\pi=\coprod_{j=1}^{k} \pi_{j}$ so that with $0 \leq a \leq b \leq k$ the condition formulated there is satisfied. Let

$$
P^{\prime}(\pi)=\left\{\mathbf{q} \in \mathbb{N}^{m}: \mathbf{q} \in P\left(\pi_{j}\right) \text { for all } j \geq b+1\right\} .
$$

Then by Corollary 4.3 there exists a constant $c_{1}>0$, depending only on $m$ and $n$, such that for any $\mathbf{q} \in P^{\prime}(\pi)$ we have

$$
N_{\pi}(\mathbf{q}) \geq c_{1}|\mathbf{q}|^{n} \prod_{j=1}^{a} \frac{\varphi\left(q_{j}\right)}{q_{j}}
$$

and in turn by $(5.5)$

$$
\lambda\left(E_{\mathbf{q}}(\mathbf{y}) \cap \mathbb{I}\right) \geq c_{2} \psi(|\mathbf{q}|)^{n} \prod_{j=1}^{a} \frac{\varphi\left(q_{j}\right)}{q_{j}}
$$

when $|\mathbf{q}|$ is sufficiently large.

Now for $\ell \in \mathbb{N}$ let

$$
S_{\ell}=\sum_{\mathbf{q} \in P^{\prime}(\pi),|\mathbf{q}|=\ell} \prod_{j=1}^{a} \frac{\varphi\left(q_{j}\right)}{q_{j}} .
$$


Then summing the two sides of the inequality (5.6) over q, we find

$$
\sum_{\mathbf{q} \in \mathbb{N}^{m}, 1 \leq|\mathbf{q}| \leq Q} \lambda\left(E_{\mathbf{q}}(\mathbf{y}) \cap \mathbb{I}\right) \geq c_{2} \sum_{\ell=Q_{0}}^{Q} S_{\ell} \psi(\ell)^{n}
$$

for all $Q \geq Q_{0}$, where $Q_{0}$ is some large integer beyond which the above lower bounds hold. Assume first that $a \geq 1$. Restricting to $\mathbf{q}$ with $q_{1}=\ell$ and $1 \leq q_{i} \leq \ell$ for all $i=2, \ldots, m$ and using Lemma 4.2, we obtain the asymptotical lower bound

$$
S_{\ell} \geq \frac{\varphi(\ell)}{\ell}\left(\sum_{j=1}^{\ell} \frac{\varphi(j)}{j}\right)^{a-1}\left(c_{3} \ell^{m-a}\right),
$$

where $c_{3}=\prod_{j=b+1}^{k} \zeta\left(d_{j}\right)^{-1}$ with $d_{j}=\operatorname{card}\left(\pi_{\mathrm{j}}\right)$. When $a=0$, we combine Lemma 4.1 for $q=Q=\ell$ with Lemma 4.2 to get the estimate $S_{\ell} \geq \varphi(\ell)\left(c_{3} \ell^{m-2}\right)$. Noting that the average value of $\varphi(j) / j$ on the interval $[1, \ell]$ is asymptotically equal to $1 / \zeta(2)$ when $\ell$ is large, we find that $S_{\ell} \geq c_{4} \varphi(\ell) \ell^{m-2}$ in any case. Hence by (5.7) we have

$$
\sum_{\mathbf{q} \in \mathbb{Z}^{m}, 1 \leq|\mathbf{q}| \leq Q} \lambda\left(E_{\mathbf{q}}(\mathbf{y}) \cap \mathbb{I}\right) \geq c_{5} \sum_{\ell=Q_{0}}^{Q} \varphi(\ell) \ell^{m-2} \psi(\ell)^{n} .
$$

Since the partial sum $\sum_{j=1}^{\ell} \frac{\varphi(j)}{j}$ is asymptotically equivalent to $\ell / \zeta(2)$ as $\ell$ tends to infinity, and the mapping $\ell \mapsto \ell^{m-1} \psi(\ell)^{n}$ is non-increasing, by Abel summation we now get that

$$
\sum_{\mathbf{q} \in \mathbb{Z}^{m}, 1 \leq|\mathbf{q}| \leq Q} \lambda\left(E_{\mathbf{q}}(\mathbf{y}) \cap \mathbb{I}\right) \geq c_{6} \sum_{\ell=Q_{0}}^{Q} \ell^{m-1} \psi(\ell)^{n} .
$$

This completes the proof of the Lemma.

Remark. In order to bound from below the volume of the intersection of an hypercube in $\mathbb{R}^{m}$ by a thickened hyperplane, we have inserted in the hypercube an euclidean ball cutting the hyperplane in an $(m-1)$-dimensional ball whose area is easily controlled. The recipe is adequate for our purpose. However, the full hyperplane section of the hypercube is much larger. Refined results could eventually be obtained using the explicit formulas of [14, 21].

\section{Completion of the proof of Theorem 5.1}

We claim that for all $\mathbf{y} \in \mathbb{R}^{n}$ we have

$$
\lambda\left(\mathcal{E}_{P(\pi)}(\psi, \mathbf{y}) \cap \mathbb{I}\right) \geq \delta:=12^{-n} 16^{-m} c^{2},
$$


where $c$ is the positive constant as in Lemma 5.3 .

Using a classical converse to the Borel-Cantelli Lemma, we obtain the lower bound

$$
\begin{aligned}
\lambda\left(\mathcal{E}_{P(\pi)}(\psi, \mathbf{y}) \cap \mathbb{I}\right) & =\lambda\left(\limsup _{|\mathbf{q}| \rightarrow+\infty} E_{\mathbf{q}}(\mathbf{y}) \cap \mathbb{I}\right) \\
& \geq \limsup _{Q \rightarrow+\infty} \frac{\left(\sum_{1 \leq|\mathbf{q}| \leq Q} \lambda\left(E_{\mathbf{q}}(\mathbf{y}) \cap \mathbb{I}\right)\right)^{2}}{\sum_{1 \leq|\mathbf{q}| \leq Q} \sum_{1 \leq\left|\mathbf{q}^{\prime}\right| \leq Q} \lambda\left(E_{\mathbf{q}}(\mathbf{y}) \cap E_{\mathbf{q}^{\prime}}(\mathbf{y}) \cap \mathbb{I}\right)} ;
\end{aligned}
$$

(see for instance Lemma 2.3 in [10]). To find a lower bound for the ratio as above we minorize its numerator and majorize the denominator. For the numerator, Lemma 5.3 provides us with the lower bound

$$
\sum_{1 \leq|\mathbf{q}| \leq Q} \lambda\left(E_{\mathbf{q}}(\mathbf{y}) \cap \mathbb{I}\right) \geq c \sum_{\ell=1}^{Q} \ell^{m-1} \psi(\ell)^{n}
$$

when $Q$ is large enough. Now consider the denominator

$$
D:=\sum_{1 \leq|\mathbf{q}| \leq Q} \sum_{1 \leq\left|\mathbf{q}^{\prime}\right| \leq Q} \lambda\left(E_{\mathbf{q}}(\mathbf{y}) \cap E_{\mathbf{q}^{\prime}}(\mathbf{y}) \cap \mathbb{I}\right) .
$$

When $\mathbf{q}$ and $\mathbf{q}^{\prime}$ are linearly independent, Lemma $5.2($ (ii) gives

$$
\lambda\left(E_{\mathbf{q}}(\mathbf{y}) \cap E_{\mathbf{q}^{\prime}}(\mathbf{y}) \cap \mathbb{I}\right) \leq 4^{n} \psi(|\mathbf{q}|)^{n} \psi\left(\left|\mathbf{q}^{\prime}\right|\right)^{n} \leq 12^{n} \psi(|\mathbf{q}|)^{n} \psi\left(\left|\mathbf{q}^{\prime}\right|\right)^{n},
$$

where the last larger term is introduced for convenience in combining with the other terms; see below. If $\mathbf{q}, \mathbf{q}^{\prime}$ are non-zero linearly dependent vectors, say with $|\mathbf{q}| \geq\left|\mathbf{q}^{\prime}\right|$, they can be uniquely written in the form $\mathbf{q}=q \mathbf{a}, \mathbf{q}^{\prime}=q^{\prime} \mathbf{a}$ with $\mathbf{a} \in \mathbb{Z}^{m} \backslash\{\mathbf{0}\}$, $q \in \mathbb{N}, q^{\prime} \in \mathbb{Z} \backslash\{0\}$, and $1 \leq\left|q^{\prime}\right| \leq q, \operatorname{gcd}\left(q, q^{\prime}\right)=1$. Then by Lemma $5.2($ iii), $\lambda\left(E_{\mathbf{q}}(\mathbf{y}) \cap E_{\mathbf{q}^{\prime}}(\mathbf{y}) \cap \mathbb{I}\right)$ is majorised by

$$
12^{n} \psi(|\mathbf{q}|)^{n} \max \left(\psi\left(\left|\mathbf{q}^{\prime}\right|\right), \frac{1}{q}\right)^{n} \leq 12^{n} \psi(|\mathbf{q}|)^{n} \psi\left(\left|\mathbf{q}^{\prime}\right|\right)^{n}+12^{n} \frac{\psi(|\mathbf{q}|)^{n}}{q^{n}} .
$$

Together with (6.3) and (6.4) this yields, on summing the terms corresponding to all the pairs involved and noting that the number of the integers $q^{\prime}$ satisfying $1 \leq\left|q^{\prime}\right| \leq q$ and $\operatorname{gcd}\left(q, q^{\prime}\right)=1$ equals $2 \varphi(q)$, that

$$
D \leq 12^{n}\left(\sum_{1 \leq|\mathbf{q}| \leq Q} \psi(|\mathbf{q}|)^{n}\right)^{2}+2 \times 12^{n} \sum_{q=1}^{Q} \frac{2 \varphi(q)}{q^{n}} \sum_{\mathbf{q} \in q \mathbb{Z}^{m}, 1 \leq|\mathbf{q}| \leq Q} \psi(|\mathbf{q}|)^{n} .
$$


Applying the obvious upper bound $4^{m}(\ell / q)^{m-1}$ for the number of elements $\mathbf{v} \in \mathbb{Z}^{m}$ with norm $|\mathbf{v}|=\ell / q$, we conclude that

$$
D \leq 12^{n} 4^{2 m}\left(\sum_{\ell=1}^{Q} \ell^{m-1} \psi(\ell)^{n}\right)^{2}+12^{n} 4^{m+1} \sum_{q=1}^{Q} \frac{\varphi(q)}{q^{n}} \sum_{q \leq \ell \leq Q, q \mid \ell}\left(\frac{\ell}{q}\right)^{m-1} \psi(\ell)^{n} .
$$

Observe now that

$$
\sum_{q \leq \ell \leq Q, q \mid \ell} \ell^{m-1} \psi(\ell)^{n} \leq q^{-1} \sum_{\ell=1}^{Q} \ell^{m-1} \psi(\ell)^{n}
$$

since the function $\ell \mapsto \ell^{m-1} \psi(\ell)^{n}$ is non-increasing. We thus obtain the bound

$$
D \leq 12^{n} 16^{m}\left(\sum_{\ell=1}^{Q} \ell^{m-1} \psi(\ell)^{n}\right)^{2}+12^{n} 4^{m+1}\left(\sum_{q=1}^{Q} \frac{\varphi(q)}{q^{m+n}}\right)\left(\sum_{\ell=1}^{Q} \ell^{m-1} \psi(\ell)^{n}\right) .
$$

Substituting from (6.2) and (6.5), we get that the right hand side term in (6.1) is at least

$$
\limsup _{Q \rightarrow+\infty} \frac{\left(c \sum_{\ell=1}^{Q} \ell^{m-1} \psi(\ell)^{n}\right)^{2}}{12^{n} 16^{m}\left(\sum_{\ell=1}^{Q} \ell^{m-1} \psi(\ell)^{n}\right)^{2}+12^{n} 4^{m+1}\left(\sum_{q=1}^{Q} \frac{\varphi(q)}{q^{m+n}}\right)\left(\sum_{\ell=1}^{Q} \ell^{m-1} \psi(\ell)^{n}\right)} .
$$

We note that since $m+n \geq 3, \sum_{q=1}^{Q} \frac{\varphi(q)}{q^{m+n}}$ is bounded, while by the condition in the hypothesis $\sum_{\ell=1}^{Q} \ell^{m-1} \psi(\ell)^{n}$ can be arbitrarily large when $Q$ is large enough. This shows that the above limsup is $12^{-n} 16^{-m} c^{2}$. Therefore $\lambda\left(\mathcal{E}_{P(\pi)}(\psi, \mathbf{y}) \cap \mathbb{I}\right) \geq$ $12^{-n} 16^{-m} c^{2}$, as claimed. This completes the proof of Theorem 5.1.

\section{$7 \quad$ Proofs of the main results}

The fact that the lower bound in Theorem 5.1 does not depend on the specific function $\psi$, so long as $x \mapsto x^{m-1} \psi(x)^{n}$ is non-increasing and the series $\sum_{\ell \geq 1} \ell^{m-1} \psi(\ell)^{n}$ diverges, is crucial to our proofs of Theorems 1.1]and 1.2 in the case when $m+n \geq 3$. We note that when the condition holds for a function $\psi$ it also holds for every scaled version $\psi_{l}, l \in \mathbb{N}$, (as in $\left.\S 2\right)$, since

$$
\sum_{j \geq 1} j^{m-1} \psi_{l}(j)^{n}=l^{-m+1} \sum_{j \geq 1}(l j)^{m-1} \psi(l j)^{n} \geq l^{-m} \sum_{j \geq l}(j)^{m-1} \psi(j)^{n} .
$$




\subsection{Proof of Theorem 1.1}

Assume first that the series $\sum_{\ell \geq 1} \ell^{m-1} \psi(\ell)^{n}$ diverges. Put

$$
\mathbb{B}=\left\{\mathbf{y} \in \mathbb{R}^{n}:|\mathbf{y}| \leq \frac{1}{2}\right\}
$$

and consider the subset $\mathcal{E}_{P(\pi)}\left(\psi_{l}\right) \cap(\mathbb{I} \times \mathbb{B})$ of $\operatorname{Mat}_{n, m}(\mathbb{R}) \times \mathbb{R}^{n}$. When $m+n \geq 3$ we get from Theorem 5.1 that there exists $\delta>0$ such that

$$
\lambda\left(\mathcal{E}_{P(\pi)}\left(\psi_{l}, \mathbf{y}\right) \cap \mathbb{I}\right) \geq \delta
$$

for all $l \in \mathbb{N}$ and $\mathbf{y} \in \mathbb{R}^{n}$. Integrating over $\mathbf{y}$ in $\mathbb{B}$, we obtain the lower bound

$$
\lambda\left(\mathcal{E}_{P(\pi)}\left(\psi_{l}\right) \cap(\mathbb{I} \times \mathbb{B})\right) \geq \delta,
$$

for all $l \in \mathbb{N}$. In the case when $m=n=1$ this statement was established in 13 (see bottom of page 422), specifically with $\delta=\frac{1}{4}$; as noted in the Introduction, in [13] Theorem 1.1 was proved for $m=n=1$ under an additional assumption that $\psi(2 \ell) \gg \psi(\ell)$; the extra assumption however is not involved in the proof of the estimate as above. Thus (7.1) holds for all $m, n \in \mathbb{N}$. This conclusion in turn implies that

$$
\lambda\left(\left(\bigcup_{\kappa \in \mathbb{N}} \mathcal{E}_{P(\pi)}\left(\kappa \psi_{l}\right)\right) \cap(\mathbb{I} \times \mathbb{B})\right) \geq \delta .
$$

Hence for $\mathcal{E}_{P(\pi)}^{\prime}(\psi)=\bigcap_{l \geq 1}\left(\bigcup_{\kappa \in \mathbb{N}} \mathcal{E}_{P(\pi)}\left(\kappa \psi_{l}\right)\right)$, the limit of the decreasing sequence of sets $\bigcup_{\kappa \in \mathbb{N}} \mathcal{E}_{P(\pi)}\left(\kappa \psi_{l}\right)$, we have

$$
\lambda\left(\mathcal{E}_{P(\pi)}^{\prime}(\psi) \cap(\mathbb{I} \times \mathbb{B})\right) \geq \delta .
$$

Thus $\mathcal{E}_{P(\pi)}^{\prime}(\psi)$ is not a null set. Therefore by Proposition 2.2 we get that it is a full set. Consequently $\bigcup_{\kappa \in \mathbb{N}} \mathcal{E}_{P(\pi)}(\kappa \psi)$ is also a full set since it contains $\mathcal{E}_{P(\pi)}^{\prime}(\psi)$. Applying Corollary 3.2 we now obtain finally that $\mathcal{E}_{P(\pi)}(\psi)$ is a full set, proving the first part of Theorem 1.1.

Assume now that the series $\sum_{\ell \geq 1} \ell^{m-1} \psi(\ell)^{n}$ converges. We have to prove that the set

$$
\begin{aligned}
F:=\left\{(\Theta, \mathbf{y}) \in \operatorname{Mat}_{n, m}(\mathbb{R}) \times \mathbb{R}^{n}:|\Theta \mathbf{q}+\mathbf{p}-\mathbf{y}| \leq \psi(|\mathbf{q}|)\right. \\
\\
\text { for infinitely many } \left.(\mathbf{q}, \mathbf{p})^{t} \in \mathbb{Z}^{m+n}\right\}
\end{aligned}
$$

is of Lebesgue measure 0. The assertion is an easy consequence of the Borel-Cantelli Lemma. The case $m=1$ is stated in Theorem II in Chapter VII of [6]. For 
completeness, here is a proof in the general case. Recalling the sets $F_{\mathbf{q}}(\mathbf{y})$ introduced in the proof of Lemma 5.2 for any $\mathbf{q} \in \mathbb{Z}^{m} \backslash\{0\}$ and any $\mathbf{y} \in \mathbb{R}^{n}$, we can write

$$
F \cap\left(\mathbb{I} \times \mathbb{R}^{n}\right)=\coprod_{\mathbf{y} \in \mathbb{R}^{n}}\left(\left\{\limsup _{\mathbf{q} \in \mathbb{Z}^{m} \backslash\{0\}} F_{\mathbf{q}}(\mathbf{y}) \cap \mathbb{I}\right\} \times\{\mathbf{y}\}\right)
$$

as a superior limit. Since $\lambda\left(F_{\mathbf{q}}(\mathbf{y}) \cap \mathbb{I}\right)=2^{n} \psi(|\mathbf{q}|)^{n}$ for any $\mathbf{y}$, we have

$$
\sum_{\mathbf{q} \in \mathbb{Z}^{m}, 1 \leq|\mathbf{q}| \leq Q} \lambda\left(F_{\mathbf{q}}(\mathbf{y}) \cap \mathbb{I}\right)=2^{n} \sum_{\mathbf{q} \in \mathbb{Z}^{m}, 1 \leq|\mathbf{q}| \leq Q} \psi(|\mathbf{q}|)^{n} \leq 4^{m} 2^{n} \sum_{\ell=1}^{Q} \ell^{m-1} \psi(\ell)^{n}
$$

by majorising the number of points $\mathbf{q} \in \mathbb{Z}^{m}$ with norm $|\mathbf{q}|=\ell$ by $4^{m} \ell^{m-1}$. Now, the Borel-Cantelli lemma yields that $\limsup F_{\mathbf{q}}(\mathbf{y}) \cap \mathbb{I}$ is a null set for every $\mathbf{y} \in \mathbb{R}^{n}$, $\mathbf{q} \in \mathbb{Z}^{m} \backslash\{0\}$

since the series $\sum_{\ell>1} \ell^{m-1} \psi(\ell)^{n}$ is convergent. Hence $F \cap\left(\mathbb{I} \times \mathbb{R}^{n}\right)$ is a null set. Observe that the subset $F$, naturally embedded in $\mathbb{R}^{m n+n}$, is stable by the group of integer translations $\mathbb{Z}^{m n+n}$. Therefore, the whole set $F$ is as well a null set. This completes the proof of Theorem 1.1.

\subsection{Proof of Theorem 1.2}

In the case $m=n=1$ Theorem 1.2 reduces to the classical Khintchine's theorem, see for instance Theorem I in Chapter VII of [6]; the constraint of coprimality can be readily met by dividing a (general) solution $(q, p)^{t}$ by $\operatorname{gcd}(p, q)$, as $\psi$ is assumed to be non-increasing. When $m+n \geq 3$ the proof of Theorem 1.2 follows along the same lines as the proof of Theorem 1.1 as above, in fact in a much simpler way, with $\mathbf{y}$ being now fixed, equal to the origin $\mathbf{0}$ in $\mathbb{R}^{n}$; in this case Proposition 2.3 plays the same role as Proposition 2.2 for Theorem 1.1. We omit the details.

\subsection{Proof of Theorem 1.3}

We distinguish the cases $\mathbf{y} \neq 0$ and $\mathbf{y}=0$. Assume first that $\mathbf{y}$ is a non-zero vector in $\mathbb{R}^{n}$. In this case, we deduce Theorem 1.3 from Theorem 1.1, the two statements are in fact equivalent. We have to show that the set $\mathcal{G}_{P(\pi)}(\psi, \mathbf{y})$ is full (resp. null) for every $\mathbf{y} \in \mathbb{R}^{n} \backslash\{0\}$ exactly when the series $\sum_{j \geq 1} j^{m-1} \psi(j)^{n}$ diverges (resp. converges). For this purpose, we relate $\mathcal{G}_{P(\pi)}(\psi, \mathbf{y})$ with the various sets

$$
\mathcal{E}_{P(\pi)}(\psi), \quad \mathcal{E}_{P(\pi)}(\psi, \mathbf{y}), \quad \mathcal{E}_{P(\pi)}(\psi, \Phi, \mathbf{y}), \quad \forall \Phi \in \operatorname{Mat}_{n, n}(\mathbb{R}), \forall \mathbf{y} \in \mathbb{R}^{n}
$$


respectively introduced in (2.3), (2.2) and in (3.1). We also set

$$
\mathcal{G}_{P(\pi)}(\psi)=\left\{((\Theta, \Phi), \mathbf{y}) \in \operatorname{Mat}_{n, m+n}(\mathbb{R}) \times \mathbb{R}^{n}:(\Theta, \Phi) \in \mathcal{G}_{P(\pi)}(\psi, \mathbf{y})\right\}
$$

Apart from these sets it would be convenient to introduce certain larger sets associated with them, consisting of the union of the corresponding sets over all $\kappa \psi$, as $\kappa$ ranges over $\mathbb{N}$; we shall denote the corresponding larger set by overlining the notation for the original set; thus, for instance $\overline{\mathcal{E}}_{P(\pi)}(\psi)=\cup_{\kappa \in \mathbb{N}} \mathcal{E}_{P(\pi)}(\kappa \psi)$. This may be compared with the sets introduced in (2.4).

Lemma 7.1. The following statements are equivalent:

(i) The set $\overline{\mathcal{E}}_{P(\pi)}(\psi)$ is full (resp. null) in $\operatorname{Mat}_{n, m}(\mathbb{R}) \times \mathbb{R}^{n}$.

(ii) There exists $\Phi \in G L(n, \mathbb{R})$ such that $\overline{\mathcal{E}}_{P(\pi)}(\psi, \Phi, \mathbf{y})$ is full (resp. null) in $\operatorname{Mat}_{n, m}(\mathbb{R})$ for almost every $\mathbf{y} \in \mathbb{R}^{n}$.

(iii) For every $\Phi \in G L(n, \mathbb{R})$ the set $\overline{\mathcal{E}}_{P(\pi)}(\psi, \Phi, \mathbf{y})$ is full (resp. null) in $\operatorname{Mat}_{n, m}(\mathbb{R})$ for almost every $\mathbf{y} \in \mathbb{R}^{n}$.

(iv) The set $\overline{\mathcal{G}}_{P(\pi)}(\psi)$ is full (resp. null) in $\operatorname{Mat}_{n, m+n}(\mathbb{R}) \times \mathbb{R}^{n}$.

(v) There exists $\mathbf{y} \in \mathbb{R}^{n} \backslash\{0\}$ such that the set $\overline{\mathcal{G}}_{P(\pi)}(\psi, \mathbf{y})$ is full (resp. null) in $\operatorname{Mat}_{n, m+n}(\mathbb{R})$.

(vi) For every $\mathbf{y} \in \mathbb{R}^{n} \backslash\{0\}$ the set $\overline{\mathcal{G}}_{P(\pi)}(\psi, \mathbf{y})$ is full (resp. null) in $\operatorname{Mat}_{n, m+n}(\mathbb{R})$.

Proof. Arguing as in the proof of Proposition 2.3, observe that the equivariance relations

$$
g \overline{\mathcal{E}}_{P(\pi)}(\psi, \Phi, \mathbf{y})=\overline{\mathcal{E}}_{P(\pi)}(\psi, g \Phi, g \mathbf{y}), \quad g \overline{\mathcal{G}}_{P(\pi)}(\psi, \mathbf{y})=\overline{\mathcal{G}}_{P(\pi)}(\psi, g \mathbf{y})
$$

hold for any $g \in G L(n, \mathbb{R}), \Phi \in \operatorname{Mat}_{n, n}(\mathbb{R}), \mathbf{y} \in \mathbb{R}^{n}$. Since the action of $G L(n, \mathbb{R})$ on $\mathbb{R}^{n} \backslash\{0\}$ is transitive, we deduce that (v) and (vi) are equivalent. Now (iv) means that $\overline{\mathcal{G}}_{P(\pi)}(\psi, \mathbf{y})$ is full (resp. null) for almost all $\mathbf{y} \in \mathbb{R}^{n}$ by the Fubini theorem. Hence (iv) is equivalent to (v) and (vi). The first equivariance relation shows in the same way that (ii) and (iii) are equivalent. Noting that for $\Phi=I d_{n}$, we have $\mathcal{E}_{P(\pi)}\left(\psi, I d_{n}, \mathbf{y}\right)=\mathcal{E}_{P(\pi)}(\psi, \mathbf{y})$, we obtain the equivalence of (i) with (ii) again by Fubini. Finally the equivalence of (iii) and (iv) follows from the obvious equality

$$
\overline{\mathcal{G}}_{P(\pi)}(\psi)=\coprod_{\Phi \in \mathrm{Mat}_{n, n}(\mathbb{R})} \coprod_{\mathbf{y} \in \mathbb{R}^{n}}\left(\overline{\mathcal{E}}_{P(\pi)}(\psi, \Phi, \mathbf{y}) \times\{\Phi\} \times\{\mathbf{y}\}\right),
$$

using Fubini, since $G L(n, \mathbb{R}) \times \mathbb{R}^{n}$ is an open set of $\operatorname{Mat}_{n, n}(\mathbb{R}) \times \mathbb{R}^{n}$.

Now, Proposition 3.1 tells us that all overlined sets occurring in Lemma 7.1 are equal, up to a null set, to the corresponding set without the bar. Thus the same 
equivalences hold true for the non-overlined sets. In particular, $\mathcal{G}_{P(\pi)}(\psi, \mathbf{y})$ is full (resp. null) if and only if $\mathcal{E}_{P(\pi)}(\psi)$ is full (resp. null). By Theorem 1.1, the latter property holds when the series $\sum_{j \geq 1} j^{m-1} \psi(j)^{n}$ diverges (resp. converges).

For the case $\mathbf{y}=\mathbf{0}$, we use the same strategy, basically multiplying on the left matrices in $\operatorname{Mat}_{n, m+n}(\mathbb{R})$ or in $\operatorname{Mat}_{n, m}(\mathbb{R})$ by $g \in G L(n, \mathbb{R})$, to relate the sets $\mathcal{G}_{P(\pi)}(\psi, \mathbf{0})$ and $\mathcal{E}_{P(\pi)}(\psi, \mathbf{0})$. The proof is simpler and we now apply Theorem 1.2. We omit the details.

Acknowledgements S.G. Dani and A. Nogueira would like to thank, respectively, the Institut de Mathématiques de Luminy, Aix-Marseille Université, France and the De Giorgi Center, Italy for hospitality while this work was done.

\section{References}

[1] M.B. Bekka and M. Mayer, Ergodic theory and topological dynamics of group actions on homogeneous spaces. London Mathematical Society Lecture Note Series, 269. Cambridge University Press, Cambridge, 2000. x+200 pp.

[2] V. Beresnevich and S. Velani, A note on zero-one laws in Diophantine approximation, Acta Arithmetica 133 (2008), 363-374.

[3] V. Beresnevitch and S. Velani, Classical metric diophantine approximation revisited : the Khintchine-Groshev theorem, Int. Math. Res. Not. IMRN 2010, 69-86.

[4] Y. Bugeaud, Approximation by algebraic numbers, Cambridge Tacts in Mathematics, 160. Cambridge University Press, 2004.

[5] Y. Bugeaud and M. Laurent, Exponents of inhomogeneous Diophantine approximation, Moscow Math. J. 5 (2005), 747-766.

[6] J. W. S. Cassels, An Introduction to Diophantine Approximation, Cambridge Tracts in Math. and Math. Phys., vol. 99, Cambridge University Press, 1957.

[7] J. W. S. Cassels, Some metrical theorems in Diophantine approximation I, Proc. Cambridge Phil. Soc. 46 (1950), 209-218.

[8] P. X. Gallagher, Metric simultaneous diophantine approximation, II, Mathematika 12 (1965), 123-127.

[9] A. Groshev, Un théorème sur les systèmes de formes linéaires, Dokl. Akad. Nauk SSSR 19 (1938), 151-152. 
[10] G. Harman, Metric Number Theory, London Mathematical Society Monographs 18, Oxford University Press, 1998.

[11] D. Y. Kleinbock and G. A. Margulis, Logarithmic laws for flows on homogeneous spaces, Invent. Math. 138 (1999), 451-494.

[12] S. Lang, Fundamentals of Diophantine Geometry, Springer-Verlag, 1983.

[13] M. Laurent and A. Nogueira, Inhomogeneous approximation with coprime integers and lattice orbits, Acta Arithmetica. 154.4 (2012), 413-427.

[14] J.-L. Marichal and M. Mossinghoff, Slices, Slabs, and Sections of the Unit hypercube, Online Journal of Analytical Combinatorics, 3 (2008).

[15] C. C. Moore, Ergodicity of flows on homogeneous spaces, Amer. J. Math. 88 (1966) 154-178.

[16] W. M. Schmidt, A metrical theorem in diophantine approximation, Canadian. J. Math. 12 (1960) 619-631.

[17] W. M. Schmidt, Metrical theorem on fractional parts of sequences, Trans. American. Math. Soc. 110 (1964) 493-518.

[18] W. M. Schmidt, Diophantine Approximation, Lecture Notes in Math., vol. 785, 1980.

[19] V. G. Sprindžuck, Metric Theory of Diophantine Approximations, V. H. Winston \& Sons, 1979.

[20] J. D. Vaaler, On the metric theory of Diophantine approximation, Pacific J. Math. 76 (1978), 527-539.

[21] J. D. Vaaler, A geometric inequality with applications to linear forms, Pacific J. Math. 83 (1979), 543-553.

SGD : Department of Mathematics, Indian Institute of Technology Bombay, Powai, Mumbai 400076, India.

sdani@math.iitb.ac.in

ML \& AN : Aix Marseille Université, CNRS, Centrale Marseille, I2M, UMR 7373, 13453 Marseille, France

michel-julien.laurent@univ-amu.fr, arnaldo.nogueira@univ-amu.fr 